\title{
Multiple metabolic phenotypes as screening criteria are correlated with the plant growth-promoting ability of rhizobacterial isolates
}

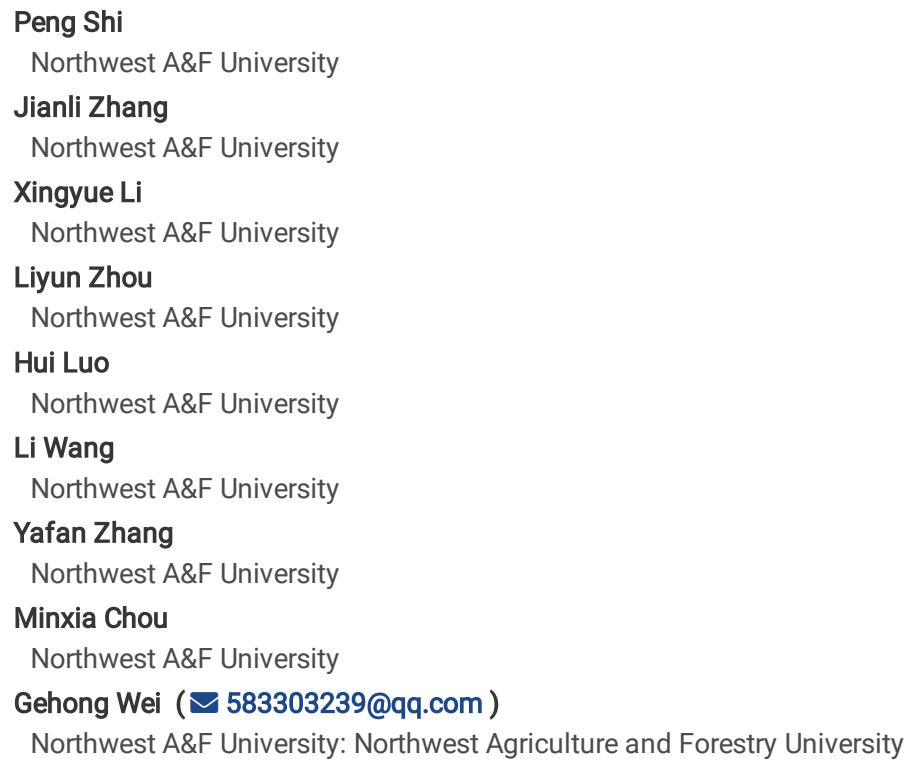

Version of Record: A version of this preprint was published at Frontiers in Microbiology on January 5th, 2022. See the published version at https://doi.org/10.3389/fmicb.2021.747982. 


\section{Abstract}

Aims

Most preliminary screening criteria for plant growth-promoting (PGP) rhizobacteria (PGPR) are based on traditional knowledge of PGP mechanisms and do not always work well due to complex plant-microbe interactions. The aim of this study was to analyze the correlation between the metabolic phenotypes of rhizobacterial isolates and their PGP ability. The feasibility of using these phenotypes as preliminary screening criteria for PGPR was also evaluated.

\section{Methods}

Twenty-one rhizobacterial isolates were screened for their PGP ability, traditional PGP traits, and multiple metabolic phenotypes that are not directly related to PGP mechanisms, but are possibly related to rhizosphere colonization. Correlations between the PGP traits or metabolic phenotypes and increases in plant agronomic parameters were analyzed to find the indicators that are most closely related to PGP ability.

\section{Results}

The utilization of 11 nutrient substrates commonly found in root exudates, such as D-salicin, $\beta$-methyl-D-glucoside, D-cellobiose, D-maltose, D-trehalose, and dextrin, was significantly positively correlated with the PGP ability of the rhizobacterial isolates. The utilization of one amino acid and two organic acids, namely L-aspartic acid, a-keto-glutaric acid, and formic acid, was negatively correlated with PGP ability. There were no significant correlations between four PGP traits tested in this study and the PGP ability.

\section{Conclusion}

The ability of rhizobacterial isolates to metabolize nutrient substrates that are identical or similar to root exudate components may act as better criteria than PGP traits for the primary screening of PGPR, because rhizosphere colonization is a prerequisite for PGPR to affect plants.

\section{Introduction}

The rhizosphere, which is the niche influenced by plant roots, is a hot spot for microbial activities in the soil (Hinsinger and Marschner 2006). Some microorganisms, termed plant growth-promoting (PGP) rhizobacteria (PGPR), colonize the rhizosphere and benefit plant growth by producing phytohormones, facilitating plant nutrient acquisition, and antagonizing plant pathogens (Ambrosini et al. 2012; Lugtenberg and Kamilova 2009; Ramos-Solano et al. 2010). At present, PGPR are regarded as an important component of biofertilizers and have great potential for application in sustainable agriculture (Backer et al. 2018; Vessey 2003).

In the natural environment, rhizosphere microorganisms interact with plants and regulate their growth via the formation of complex communities (Chaney and Baucomn 2020). In order to use PGPR as artificial inoculants in practice, it is necessary to isolate pure strains. However, there is a huge number and great diversity of microorganisms that colonize the rhizosphere, and new culture techniques allow us to obtain thousands of isolates simultaneously from the environment. Therefore, it remains challenging to identify a few key strains with the best PGP performance by screening a large number of isolates obtained from the rhizosphere niche (Bai et al. 2015; Ling et al. 2015). In addition, the performance of PGPR may be affected by climatic and environmental conditions, including soil type, temperature, plant cultivar, and interactions with other microorganisms. The data from field trials therefore provide the most accurate basis for screening the best PGPR strains for application in agriculture (Tabassum et al. 2017). Despite this, the large time requirements and high cost of field trials limit their application for large-scale screening of PGPR strains (Moretti et al. 2020).

Many studies have screened for PGPR using a relatively efficient protocol involving the following three steps (Kumar et al. 2015; Soldan et al. 2019; VasseurCoronado et al. 2020). The first step is preliminary screening: a small proportion of candidate strains that are most likely to be PGPR are selected, based on the screening of some PGP traits in vitro, from hundreds or even thousands of randomly obtained isolates. The second step is evaluation in simulated "real environmental conditions": some strains exhibiting a PGP ability are screened out by evaluating their effects on plant growth in a greenhouse with sterile substrate or real soil. The last step is field evaluation: several strains with the best PGP ability are identified based on practical application. Because the results of greenhouse and field trials provide direct evidence for the PGP ability of the strains, these data are meaningful for the screening of PGPR strains. However, for the overall screening process, it may be more important to improve the accuracy and efficiency of the in vitro preliminary screening, because most of the candidates (usually $>90 \%$ ) are eliminated in this step (Di Benedetto et al. 2019; Nordstedt and Jones 2020).

Most PGP traits that are currently used as key indicators in the preliminary screening of PGPR are designed based on known PGP mechanisms, such as solubilizing phosphate and producing siderophores. However, these indicators may not work as well in practice as theoretically predicted due to the variety of PGP mechanisms, the differences between rhizosphere and in vitro environments, and the functional redundancy of rhizosphere microorganisms (Canto et al. 2020; Finkel et al. 2017). Many studies have shown a lack of connection between these PGP traits and the actual PGP ability of candidate strains (Backer et al. 2018; Cattelan et al. 1999). For example, Rajendran et al. (2008) screened out three PGPR strains that cannot solubilize phosphates but can produce siderophores. Meanwhile, Zhang et al. (2017) reported three PGPR strains with high phosphate-solubilizing ability that cannot produce siderophores. These phenomena suggest that PGP traits may not be the best criteria for the preliminary screening of PGPR; more universal and effective indicators should be identified. 
As members of the rhizosphere microbiome, PGPR are involved in complex interactions with plants that enable them to colonize close to the root surface (Backer et al. 2018; Lami et al. 2020). There is increasing evidence indicating that the phenotypes of PGPR strains associated with these interactions (such as production of plant polymer-degrading enzymes and biofilm formation) are essential for their adaptation to the rhizosphere niche, and may be markers of PGPR (Shi et al. 2012; Valetti et al. 2018; Walitang et al. 2017; Yuan et al. 2015). Therefore, it may be reasonable to use phenotypes related to plant-microbe interactions as preliminary screening criteria for PGPR, given that successful colonization is the key prerequisite for PGPR to act on plants.

Root exudates, which are composed of sugars, amino acids, and organic acids, play an active role in the interactions between plants and rhizosphere microorganisms. Root exudates provide rhizosphere microorganisms with nutrient sources and serve as interacting signals (Badri and Vivanco 2009; Bais et al. 2006; Canto et al. 2020; Hassan et al. 2019; Shi et al. 2012; Yuan et al. 2015). The capacity of rhizosphere microorganisms to utilize the nutrients in root exudates is a vital metabolic feature, both for PGPR and pathogens (Pascale et al. 2020; Rodriguez et al. 2019). Many studies have explored on the nutrient utilization capacities of plant pathogens and these capacities have been regarded as an indicator of pathogenicity or for biological control evaluation (Huang et al. 2020; Irikiin et al. 2006; Ji and Wilson 2002; Jian et al. 2019; Zhang et al. 2019). However, the nutrient utilization capacities of PGPR have not been paid enough attention, and the possibility of using these capacities as preliminary screening indicators has rarely been reported.

In legumes, the root nodule is a specialized organ that plays a pivotal role in nitrogen supply. In the case of the soybean-rhizobium symbiosis, PGPR can promote both host plant growth (Moretti et al. 2020) and rhizobium symbiotic nodulation (Han et al. 2020). This symbiosis system may be an ideal model to assess the accuracy and universality of PGPR prescreening indicators because it can be affected by microorganisms via more diverse mechanisms than the single plant system. Thus, in the present study, the soybean-rhizobium symbiosis system was used to evaluate the correlations between multiple nutrient utilization phenotypes of rhizobacteria and their PGP ability. Further, the feasibility of using these phenotypes as the main indicators for the pre-screening of PGPR was explored.

\section{Materials And Methods}

Sampling and soil characterization

Using a trowel, several individual plants of the soybean (Glycine max [L.] Merrill) cultivar Zhonghuang 13 were uprooted randomly from a field in Yangling, Shaanxi Province, China $\left(34^{\circ} 16^{\prime} \mathrm{N}, 108^{\circ} 4^{\prime} \mathrm{E}\right)$. The plant sampling was carried out during the flowering stage of soybean in June 2009 . Three of the plants with the best growth (each had more than 50 large red nodules) were immediately transported to the laboratory for bacterial isolation. The soil (Lou soil) was also sampled from the same field, and the basic soil characteristics were analyzed at the Test Center of Northwest A\&F University (Yangling, China). The soil had a $\mathrm{pH}$ of 7.84 and contained a total $\mathrm{N}$ content of $980 \mathrm{mg} \mathrm{kg}^{-1}$, available $\mathrm{N}$ of $10.83 \mathrm{mg} \mathrm{kg}^{-1}$, total P of $1 \mathrm{~g} \mathrm{~kg}^{-1}$, available P of $6.48 \mathrm{mg} \mathrm{kg}$, and available Fe of $9.4 \mathrm{mg} \mathrm{kg}^{-1}$.

Rhizobacterial and rhizobial isolation

Rhizobacteria were isolated from the surface of root nodules according to the method described by Kuklinsky-Sobral et al. (2004). Briefly, after the bulk soil was removed, all red nodules were picked off the roots using aseptic forceps. The nodules were washed using sterile water three times to remove the bacterial cells that were not firmly adhered to the nodule surface. Then, the nodules were placed in Erlenmeyer flasks containing glass beads and saline solution ( $0.7 \%$ $\mathrm{NaCl})$. The flasks were shaken at $150 \mathrm{rpm}$ for $1 \mathrm{~h}$ at $28^{\circ} \mathrm{C}$. After agitation, an appropriate dilution was plated onto nutrient agar plates (ATCC $₫$ Medium 3 ) and the plates were incubated at $28^{\circ} \mathrm{C}$ for $2-7 \mathrm{~d}$. Visually different colonies were selected and purified by repeatedly streaking.

After isolating the rhizobacteria, the nodules were immediately further used to isolate rhizobia. The nodules were surface sterilized with $75 \%$ ( $/ \mathrm{v}$ ) alcohol for $30 \mathrm{~s}$, followed by $1 \%(\mathrm{w} / \mathrm{v})$ sodium hypochlorite for $4 \mathrm{~min}$, and then rinsed six times with sterile distilled water. Subsequently, 59 large red nodules (a quarter of the total nodules) were crushed with aseptic forceps and streaked onto yeast mannitol agar plates (Vincent 1970 ); the plates were incubated at $28^{\circ} \mathrm{C}$ for $3-15$ d. Rhizobial colonies were purified by repeatedly streaking and were checked via observation of the colony and cellular morphology and nodulation tests (Vincent 1970). The isolate that was found in the largest number of nodules was selected for co-inoculation experiments.

Rhizobacterial and rhizobial characterization

All rhizobacterial and rhizobial isolates were screened for their PGP traits. Mineral phosphate-solubilizing activity was measured using agar plates with $10.0 \mathrm{~g}$ $\mathrm{L}^{-1} \mathrm{Ca}_{3}\left(\mathrm{PO}_{4}\right)_{2}$ according to Qin et al. (2011). Chitinase activity was measured using agar plates with $15.0 \mathrm{~g} \mathrm{~L}^{-1}$ colloidal chitin according to Monreal and Reese (1969). Siderophore production was tested using chrome azurol S agar plates using the method described by Schwyn and Neidlands (1987). All isolates were incubated at $28^{\circ} \mathrm{C}$. The ratio of the diameter of the clear zone (halo) or color halo surrounding the colony to the diameter of the colony was used to evaluate the ability of the isolates to solubilize phosphate and produce chitinase and siderophores (Kobayashi et al. 1995; Kumar et al. 2012; Marra et al. 2012; Rau et al. 2009; Suresh 2012). The indole acetic acid (IAA) production was tested using the method described by Glickmann and Dessaux (1995). Briefly, the isolates were cultured in King's B medium with agitation at $150 \mathrm{rpm}$ for $7 \mathrm{~d}$ at $28^{\circ} \mathrm{C}$. The cultures were centrifuged at $9600 \times g$ for $10 \mathrm{~min}$. The supernatant was mixed with Salkowski reagent $(1: 1 \mathrm{v} / \mathrm{v})$ and placed in darkness for $30 \mathrm{~min}$. The optical density was read at $530 \mathrm{~nm}$ on a LAMBDA $35 \mathrm{UV} / \mathrm{Vis}$ spectrophotometer (PerkinElmer, Shelton, CT, USA). The IAA concentration was calculated according to the IAA standard curve (0-100 mg L-1).

All rhizobacterial and rhizobial isolates were identified by sequencing the $16 \mathrm{~S}$ rRNA gene. The rhizobacterial and rhizobial isolates were grown in $5 \mathrm{~mL}$ of tryptone yeast extract broth (Sharma et al. 2009) and yeast mannitol broth (Vincent 1970), respectively, at $28^{\circ} \mathrm{C}$ for $2 \mathrm{~d}$ with agitation (150 rpm). Then, genomic DNA was extracted with the method described by Zhao et al. (2010) and used as the template. The full-length 16S rRNA gene was amplified using the primers P1 (5'-CGGGATCCAGAGTTTGATCCTGGTCAGAACGAACGCT-3) and P6 (5'-CGGGATCCTACGGCTACCTTGTTACGACTTCACCCC-3) (Zhao et al. 2010). The PCR products were digested separately using the restriction endonucleases Hhal, Haell, and Hinfl with the method described by Zhao et al. (2010). 
The restricted fragments were analyzed using $2 \%(\mathrm{w} / \mathrm{v})$ agarose gel electrophoresis. Isolates sharing identical restriction fragment length polymorphism patterns were defined as the same 16S rRNA genotype. The 16S rRNA genes of representative isolates with different 16S rRNA genotypes were sequenced and aligned using the EzBioCloud Database (http://www.ezbiocloud.net).

Based on the results of the PGP traits screening and 16S rRNA gene sequence alignment, 21 rhizobacterial isolates were selected for a single inoculation experiment. The single inoculation experiment was carried out before co-inoculation experiments to examine the effects of the selected rhizobacterial isolates on soybean plants without the rhizobial symbiont. Seeds of soybean cultivar Zhonghuang 13 were sorted for uniformity, and were surface sterilized with $75 \%$ $(\mathrm{v} / \mathrm{v})$ alcohol and $1 \%(\mathrm{w} / \mathrm{v})$ sodium hypochlorite. The seeds were then germinated on $1.2 \%(\mathrm{w} / \mathrm{v})$ water agar plates at room temperature in the dark for $3 \mathrm{~d}($ Fox et al. 2011). The seedlings were then planted in pots filled with a sterilized vermiculite-perlite mixture (2:1, v/v). The rhizobacterial isolates were cultured in tryptone yeast extract broth (Sharma et al. 2009) and prepared in cell suspensions (optical density of 0.55 at $600 \mathrm{~nm}$, approximately $10^{8}-10^{9}$ cells $\mathrm{mL}^{-1}$ ). The cell suspensions were injected into the rhizosphere of the soybean plants when the cotyledons had unfolded. The plants were cultivated in a greenhouse and harvested at 30 days post-inoculation (dpi) to measure the dry weights of the roots and shoots and the number of root nodules. The ratio of the dry weight of the inoculated plants (nine replicates) to that of the non-inoculated plants (24 replicates) was used to assess the effects of the isolate on the plant.

Metabolic phenotype analysis was carried out for the 21 rhizobacterial isolates using the Biolog ${ }^{\text {TM }}$ GenIII ID system (Biolog, Hayward, CA, USA) according to protocol A provided by the manufacturer. Briefly, isolates were incubated on nutrient agar plates (ATCC $\AA$ Medium 3 ) for $24 \mathrm{~h}$ at $28^{\circ} \mathrm{C}$. All isolates (two replicates per isolate) were then inoculated into inoculation fluid A (IF-A, Biolog, Hayward, CA, USA) to give an optical density of $95 \%$. Of the cell suspensions, $100 \mu \mathrm{L}$ was inoculated into each well of a GENIII microplate. The plates were incubated at $28^{\circ} \mathrm{C}$, and absorbance data were recorded at 12,24 , and $36 \mathrm{~h}$ using the Biolog station ELx808BLG (Biolog, Hayward, CA, USA) (Van Assche et al. 2017). The data collected at $36 \mathrm{~h}$ were used in the subsequent analysis because the readings of the two replicates were most consistent (Wozniak et al. 2019). The Well Color Development (WCD; end-point absorbance value of each well the negative control well value; set to zero if the value was a small negative number) value was used to evaluate the ability of an isolate to utilize nutrient substrates (two replicates for one nutrient substrate).

\section{Co-inoculation experiments}

To verify whether the effects of the rhizobacterial isolates on the soybean-rhizobium symbiosis were stable, co-inoculation experiments were performed three times over two different years (2012 and 2014). In the first experiment, which was conducted in 2012, the 21 isolates were randomly divided into six groups: (1) CCNWSP2, CCNWSP26, and CCNWSP31; (2) CCNWSP33, CCNWSP68, CCNWSP76, and CCNWSP78; (3) CCNWSP27, CCNWSP30, and CCNWSP60; (4) CCNWSP11, CCNWSP13-2, CCNWSP25, and CCNWSP46; (5) CCNWSP10, CCNWSP15, and CCNWSP21; and (6) CCNWSP4, CCNWSP13-4, CCNWSP21-1, and CCNWSP92. The co-inoculation treatment involved CCNWSX1528 and a rhizobacterial isolate, whereas the control treatment involved the rhizobial isolate CCNWSX1528 alone. For each treatment, three replicates (five plants per replicate) were included. The second and third experiments were performed in 2014 by two different researchers at the same time. The treatments were the same as those used for the experiment conducted in 2012 , with three replicates (three plants per replicate) per treatment.

During each experiment, seeds of soybean cultivar Zhonghuang 13 were surface sterilized and germinated as performed in the inoculation experiment (Fox et al. 2011). The seedlings were then planted in $1 \mathrm{~L}$ pots (one seedling per pot) filled with a sterilized vermiculite-perlite mixture (2:1, v/v). The seedlings were watered with $150 \mathrm{~mL}$ of nitrogen-free plant nutrient solution containing the following ingredients: $0.5 \mathrm{~g} \mathrm{~L}^{-1} \mathrm{~K}_{2} \mathrm{HPO}_{4}, 2.0 \mathrm{~g} \mathrm{~L}^{-1} \mathrm{Ca}_{3}\left(\mathrm{PO}_{4}\right)_{2}, 0.2 \mathrm{~g} \mathrm{~L}^{-1}$ $\mathrm{MgSO}_{4} \cdot 7 \mathrm{H}_{2} \mathrm{O}, 0.1 \mathrm{~g} \mathrm{~L}^{-1} \mathrm{NaCl}$, and $0.01 \mathrm{~g} \mathrm{~L}^{-1} \mathrm{FeCl}_{3}$ (Vincent 1970). All pots were placed in a greenhouse under a $14 / 10 \mathrm{~h}$ light/dark cycle and $25 / 20^{\circ} \mathrm{C}$ day/night temperatures.

The rhizobial isolate, CCNWSX1528, was cultured in yeast mannitol broth (Vincent 1970) for $3 \mathrm{~d}$, and the rhizobacterial isolates were cultured in tryptone yeast extract broth (Sharma et al. 2009) for $2 \mathrm{~d}$. Both types of isolate were cultured at $28^{\circ} \mathrm{C}$ with agitation (150 rpm). Cultures were centrifuged at $1600 \times g$ for 10 min, and the cell pellets were suspended in $\mathrm{NaCl}$ solution $(0.7 \% \mathrm{w} / \mathrm{v})$ to an optical density of 0.55 at $600 \mathrm{~nm}$ (approximately $10^{8}-10^{9} \mathrm{cells}^{\mathrm{mL}} \mathrm{H}^{-1}$ ). When the soybean cotyledons had unfolded, $150 \mathrm{~mL}$ of nitrogen-free plant nutrient solution and one of the following inoculants were injected into the rhizosphere: (1) 1 $\mathrm{mL}$ of $0.7 \% \mathrm{NaCl}$ solution and $1 \mathrm{~mL}$ of CCNWSX1528 suspension for the control treatment; and (2) $1 \mathrm{~mL}$ of rhizobacterial suspension and $1 \mathrm{~mL}$ of CCNWSX1528 suspension for the co-inoculation treatment. The plants were cultivated in a greenhouse, watered with $150 \mathrm{~mL}$ of nitrogen-free plant nutrient solution every $10 \mathrm{~d}$, and harvested at $30 \mathrm{dpi}$.

All five (first experiment) or three (second and third experiments) plants in each replicate were pooled to measure the growth and symbiotic nodulation of the plants. The roots, shoots, and root nodules were dried for $4 \mathrm{~d}$ at $80^{\circ} \mathrm{C}$ to measure the root dry weight (RDW), shoot dry weight (SDW), and dry weight of total nodules per plant. The total number of nodules (TNN) and the number of red nodules (RNN) per plant were recorded (Egamberdieva et al. 2013; Fox et al. 2011). The dry weight per nodule (NDW) was calculated as the ratio of the dry weight of total nodules per plant to TNN. The root total nitrogen (RN) and shoot total nitrogen (SN) per plant were analyzed using an automatic Kjeldahl nitrogen analyzer (KJEL-AUTO VS-KT-P; MRK, Tokyo, Japan).

Statistical analysis

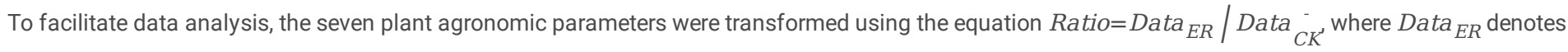
the data of each replicate in the co-inoculation treatments, and Data $_{C K}$ denotes the mean of the data in the control treatment. The mean Ratio value of the seven parameters was calculated as a comprehensive index $(\mathrm{Cl})$ and used to indicate the total effects of the rhizobacterial isolates on the soybean-rhizobium symbiosis system. The significance of differences between treatments was determined via one-way analysis of variance (ANOVA) and Dunnett's tests. Spearman's correlation coefficients were used to assess the relationships between multiple phenotypes of rhizobacterial isolates and their PGP ability. All statistical analyses were carried out using IBM SPSS Statistics 22 (IBM Corp., Armonk, NY, USA).

Page 4/21 


\section{Results}

Isolation and characterization of rhizobia and rhizobacteria

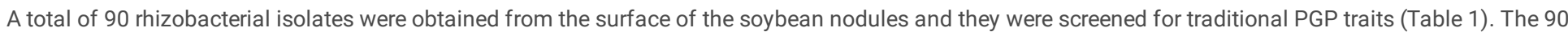

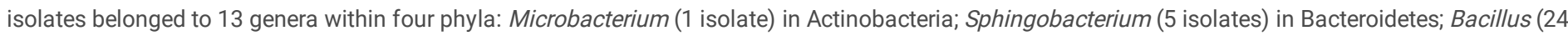

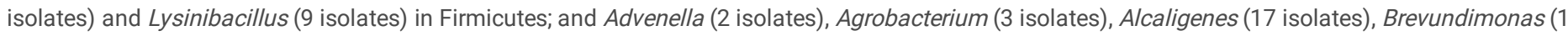
isolate), Paracoccus (2 isolates), Pseudochrobactrum (16 isolates), Shinella (1 isolate), Ensifer (5 isolates), and Stenotrophomonas (4 isolates) in

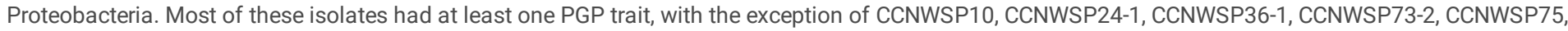

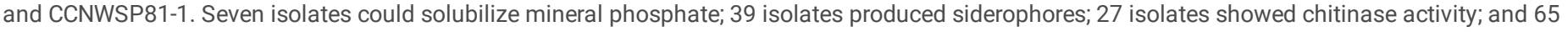
isolates produced IAA. 
Table 1

Rhizobacteria isolated in this study and their PGP traits

\begin{tabular}{|c|c|c|c|c|c|}
\hline \multirow[t]{2}{*}{ Isolates } & \multicolumn{4}{|l|}{ PGPR characteristics } & \multirow{2}{*}{$\begin{array}{l}\text { Number of } \\
\text { isolates }\end{array}$} \\
\hline & $\begin{array}{l}\text { Mineral phosphate } \\
\text { solubilization }\end{array}$ & $\begin{array}{l}\text { Siderophore } \\
\text { production }\end{array}$ & $\begin{array}{l}\text { Chitinase } \\
\text { production }\end{array}$ & $\begin{array}{l}\text { Indole acetic acid } \\
\text { production }\end{array}$ & \\
\hline \multicolumn{5}{|l|}{ Microbacterium sp. } & \multirow[t]{2}{*}{1} \\
\hline CCNWSP60 & - & - & - & प & \\
\hline \multicolumn{5}{|l|}{ Sphingobacterium sp. } & \multirow[t]{4}{*}{5} \\
\hline CCNWSP24-1,CCNWSP36-1, CCNWSP81-1 & - & - & - & - & \\
\hline CCNWSP31 & - & प & - & - & \\
\hline CCNWSP93-2 & - & - & - & प & \\
\hline \multicolumn{5}{|l|}{ Bacillus sp. } & \multirow[t]{10}{*}{24} \\
\hline $\begin{array}{l}\text { CCNWSP9, CCNWSP11, CCNWSP16, } \\
\text { CCNWSP22, }\end{array}$ & - & - & प & प & \\
\hline \multicolumn{5}{|l|}{$\begin{array}{l}\text { CCNWSP29-1, CCNWSP34, CCNWSP35-1, } \\
\text { CCNWSP37, }\end{array}$} & \\
\hline \multicolumn{5}{|l|}{$\begin{array}{l}\text { CCNWSP46, CCNWSP52, CCNWSP56, } \\
\text { CCNWSP57, }\end{array}$} & \\
\hline \multicolumn{5}{|l|}{$\begin{array}{l}\text { CCNWSP64, CCNWSP69, CCNWSP77, } \\
\text { CCNWSP79, }\end{array}$} & \\
\hline \multicolumn{5}{|l|}{ CCNWSP84, CCNWSP88,CCNWSP99 } & \\
\hline CCNWSP2, CCNWSP76 & - & - & प & - & \\
\hline CCNWSP14 & - & - & - & प & \\
\hline CCNWSP75 & - & - & - & - & \\
\hline CCNWSP1 & - & प & प & प & \\
\hline \multicolumn{5}{|l|}{ Lysinibacillus sp. } & \multirow[t]{4}{*}{9} \\
\hline $\begin{array}{l}\text { CCNWSP21, CCNWSP35, CCNWSP36, } \\
\text { CCNWSP89, }\end{array}$ & - & - & - & प & \\
\hline \multicolumn{5}{|l|}{$\begin{array}{l}\text { CCNWSP90, CCNWSP91, CCNWSP96, } \\
\text { CCNWSP102 }\end{array}$} & \\
\hline CCNWSP63 & प & - & - & प & \\
\hline \multicolumn{5}{|l|}{ Advenella sp. } & \multirow[t]{2}{*}{2} \\
\hline CCNWSP27-2, CCNWSP33 & प & $\square$ & - & $\square$ & \\
\hline \multicolumn{5}{|l|}{ Agrobacterium sp. } & \multirow[t]{3}{*}{3} \\
\hline CCNWSP2-1, CCNWSP26 & - & - & - & $\square$ & \\
\hline CCNWSP100 & - & 口 & - & प & \\
\hline \multicolumn{5}{|l|}{ Alcaligenes sp. } & \multirow[t]{6}{*}{17} \\
\hline $\begin{array}{l}\text { CCNWSP6, CCNWSP13, CCNWSP13-2, } \\
\text { CCNWSP13-4, }\end{array}$ & - & प & - & - & \\
\hline \multicolumn{5}{|l|}{$\begin{array}{l}\text { CCNWSP30, CCNWSP34-1, CCNWSP35-3, } \\
\text { CCNWSP48, }\end{array}$} & \\
\hline \multicolumn{5}{|l|}{$\begin{array}{l}\text { CCNWSP60-1, CCNWSP65-1, CCNWSP76-1, } \\
\text { CCNWSP78, }\end{array}$} & \\
\hline \multicolumn{5}{|l|}{$\begin{array}{l}\text { CCNWSP81, CCNWSP90-2, CCNWSP93, } \\
\text { CCNWSP93-1 }\end{array}$} & \\
\hline CCNWSP73-2 & - & - & - & - & \\
\hline
\end{tabular}

$\nabla$ Positive for the trait

- Negative for the trait 
CCNWSP10

Paracoccus sp.

CCNWSP6-1, CCNWSP27

$-$

$-$

[

Pseudochrobactrum sp.

CCNWSP4, CCNWSP11-2, CCNWSP21-1, CCNWSP23,

CCNWSP29-2, CNWSP29-3, CCNWSP35-2,

CCNWSP59-3,

CCNWSP65,CCNWSP65-2, CCNWSP68,

CCNWSP73,

CCNWSP81-2, CNWSP85-1,CCNWSP90-4

CCNWSP25

-

Shinella sp.

CCNWSP92

Ensifer (Sinorhizobium) sp. CCNWSP17, CCNWSP19-1,CCNWSP20, CCNWSP27-1

CCNWSP32

Stenotrophomonas sp.

$-$

口

$-$

[ $-$

CCNWSP15, CCNWSP47-1,CCNWSP83

CCNWSP72

$\otimes$ Positive for the trait

- Negative for the trait

A total of 126 rhizobial isolates were recovered from the soybean nodules. One of these, namely CCNWSX1528, showed a strong nodulation ability in the nodulation test (28 nodules per plant on average, $n=3$ ), and was therefore chosen for plant co-inoculation experiments. The 16S rRNA gene sequence of this isolate (accession number KF735789) showed $99.32 \%$ and $99.13 \%$ similarity with the sequences of Ensifer shofinae CCBAU $251167^{\top}$ and Ensifer fredii NBRC $14780^{\top}$, respectively. This isolate could produce IAA and solubilize mineral phosphate in vitro, but did not produce siderophores or chitinase (Table 2 ). 
Table 2

The PGP traits and closest relatives of the rhizobial and rhizobacterial isolates used for the inoculation experiments

\begin{tabular}{|c|c|c|c|c|c|c|c|c|c|}
\hline Phylum & Genus & Isolates & $\begin{array}{l}\text { Mineral } \\
\text { phosphate } \\
\text { solubilization }^{a}\end{array}$ & $\begin{array}{l}\text { Siderophore } \\
\text { production }^{\mathrm{a}}\end{array}$ & $\begin{array}{l}\text { Chitinase } \\
\text { production }^{a}\end{array}$ & $\begin{array}{l}\text { Indole } \\
\text { acetic acid } \\
\text { production } \\
\left(\mathrm{mg} \mathrm{L}^{-1}\right)\end{array}$ & $\begin{array}{l}\text { Strain } \\
\text { preservation } \\
\text { number }\end{array}$ & $\begin{array}{l}\text { GenBank } \\
\text { accession } \\
\text { number of } \\
16 S \text { rDNA } \\
\text { sequence }\end{array}$ & $\begin{array}{l}\text { Clos } \\
\text { (Seq } \\
\text { simi }\end{array}$ \\
\hline Proteobacteria & Ensifer & CCNWSX1528 & $2.10 \pm 0.17$ & $-b$ & - & $\begin{array}{l}51.36 \pm \\
4.13\end{array}$ & ACCC19832 & KF735789 & $\begin{array}{l}\text { Ensi } \\
\text { (Sinc } \\
\text { shot } \\
2511\end{array}$ \\
\hline Actinobacteria & Microbacterium & CCNWSP60 & - & - & - & $\begin{array}{l}13.27 \pm \\
0.23\end{array}$ & ACCC19329 & KF735798 & $\begin{array}{l}\text { Micr } \\
\text { shá́ }\end{array}$ \\
\hline & & & & & & & & & $\begin{array}{l}\text { CCN } \\
(100\end{array}$ \\
\hline Bacteroidetes & Sphingobacterium & CCNWSP31 & - & $1.41 \pm 0.08$ & - & - & ACCC19831 & KF735791 & $\begin{array}{l}\text { Sphi } \\
\text { yank }\end{array}$ \\
\hline & & & & & & & & & $\begin{array}{l}\text { CCN } \\
(100\end{array}$ \\
\hline \multirow[t]{5}{*}{ Firmicutes } & \multirow[t]{4}{*}{ Bacillus } & CCNWSP2 & - & - & $1.39 \pm 0.02$ & - & ACCC19813 & KF735790 & $\mathrm{Baci}$ \\
\hline & & CCNWSP11 & - & - & $1.37 \pm 0.06$ & $\begin{array}{l}14.47 \pm \\
0.35\end{array}$ & ACCC19814 & KF735802 & $\begin{array}{l}\mathrm{BaCl} \\
\text { ATCI } \\
\text { (98.\& }\end{array}$ \\
\hline & & CCNWSP46 & - & - & $1.43 \pm 0.09$ & $\begin{array}{l}14.73 \pm \\
0.84\end{array}$ & ACCC19811 & KF735801 & \\
\hline & & CCNWSP76 & - & - & $1.14 \pm 0.03$ & - & ACCC19812 & KF735793 & $\mathrm{Baci}$ \\
\hline & Lysinibacillus & CCNWSP21 & - & - & - & $\begin{array}{l}33.83 \pm \\
2.83\end{array}$ & ACCC19816 & KF735803 & $\begin{array}{l}\text { Lysii } \\
\text { fusii } \\
1571\end{array}$ \\
\hline \multirow[t]{11}{*}{ Proteobacteria } & Advenella & CCNWSP33 & $2.66 \pm 0.32$ & $2.78 \pm 0.28$ & - & $\begin{array}{l}16.93 \pm \\
0.90\end{array}$ & ACCC19825 & KF735794 & $\begin{array}{l}\text { Adve } \\
\text { kast } \\
\text { subs } \\
\text { PK1 }\end{array}$ \\
\hline & Agrobacterium & CCNWSP26 & - & - & - & $\begin{array}{l}22.13 \pm \\
0.64\end{array}$ & ACCC19822 & KF735792 & $\begin{array}{l}\text { Agrc } \\
\text { deltc } \\
(100\end{array}$ \\
\hline & \multirow[t]{8}{*}{ Alcaligenes } & CCNWSP13-2 & - & $1.28 \pm 0.01$ & - & - & ACCC19828 & KF735810 & $\begin{array}{l}\text { Alca } \\
\text { subs } \\
\text { DSN }\end{array}$ \\
\hline & & & & & & & & & $165 c$ \\
\hline & & CCNWSP13-4 & - & $1.55 \pm 0.07$ & - & - & ACCC19827 & KF735809 & $\begin{array}{l}\text { Alca } \\
\text { subs } \\
\text { DSN }\end{array}$ \\
\hline & & & & & & & & & $165 c$ \\
\hline & & CCNWSP30 & - & $1.87 \pm 0.07$ & - & - & ACCC19829 & KF735799 & $\begin{array}{l}\text { Alca } \\
\text { subs }\end{array}$ \\
\hline & & & & & & & & & $\begin{array}{l}\text { DSN } \\
(99.4\end{array}$ \\
\hline & & CCNWSP78 & - & $1.97 \pm 0.17$ & - & - & ACCC19826 & KF735797 & $\begin{array}{l}\text { Alca } \\
\text { subs }\end{array}$ \\
\hline & & & & & & & & & $\begin{array}{l}\text { DSN } \\
(100\end{array}$ \\
\hline & Brevundimonas & CCNWSP10 & - & - & - & - & ACCC19824 & KF735808 & $\begin{array}{l}\text { Brev } \\
\text { bullic } \\
(99.4\end{array}$ \\
\hline
\end{tabular}

a The ratio of the halo diameter to the colony diameter was used to indicate the ability of an isolate to solubilize mineral phosphate and produce siderophore Data are presented as means \pm standard errors $(n=3)$.

b - Negative for the trait. 


\begin{tabular}{|c|c|c|c|c|c|c|c|c|c|}
\hline Phylum & Genus & Isolates & $\begin{array}{l}\text { Mineral } \\
\text { phosphate } \\
\text { solubilization }\end{array}$ & $\begin{array}{l}\text { Siderophore } \\
\text { production }^{\mathrm{a}}\end{array}$ & $\begin{array}{l}\text { Chitinase } \\
\text { production }^{\mathrm{a}}\end{array}$ & $\begin{array}{l}\text { Indole } \\
\text { acetic acid } \\
\text { production } \\
\left(\mathrm{mg} \mathrm{L}^{-1}\right)\end{array}$ & $\begin{array}{l}\text { Strain } \\
\text { preservation } \\
\text { number }\end{array}$ & $\begin{array}{l}\text { GenBank } \\
\text { accession } \\
\text { number of } \\
16 S \text { rDNA } \\
\text { sequence }\end{array}$ & $\begin{array}{l}\text { Clos } \\
\text { (Seq } \\
\text { simi }\end{array}$ \\
\hline & Paracoccus & CCNWSP27 & - & - & - & $\begin{array}{l}58.90 \pm \\
6.33\end{array}$ & ACCC19823 & KF735800 & $\begin{array}{l}\text { Pará } \\
\text { litori } \\
05^{\top} \text {, }\end{array}$ \\
\hline & \multirow[t]{8}{*}{ Pseudochrobactrum } & CCNWSP4 & - & $1.42 \pm 0.08$ & - & $\begin{array}{l}69.60 \pm \\
6.67\end{array}$ & ACCC19820 & KF735806 & $\begin{array}{l}\text { Psel } \\
\text { asac }\end{array}$ \\
\hline & & & & & & & & & \\
\hline & & CCNWSP21-1 & - & $1.50 \pm 0.03$ & - & $\begin{array}{l}391.5 \pm \\
7.8\end{array}$ & ACCC19819 & KF735805 & $\begin{array}{l}\text { Psel } \\
\text { asac } \\
\text { DSN }\end{array}$ \\
\hline & & & & & & & & & \\
\hline & & CCNWSP25 & - & - & $1.70 \pm 0.23$ & $\begin{array}{l}28.93 \pm \\
7.20\end{array}$ & ACCC19818 & KF735804 & $\begin{array}{l}\text { Psel } \\
\text { asac }\end{array}$ \\
\hline & & & & & & & & & $\begin{array}{l}\text { DSN } \\
(99.2\end{array}$ \\
\hline & & CCNWSP68 & - & $1.97 \pm 0.28$ & - & $\begin{array}{l}345.2 \pm \\
31.3\end{array}$ & ACCC19817 & KF735796 & $\begin{array}{l}\text { Psel } \\
\text { asac }\end{array}$ \\
\hline & & & & & & & & & $\begin{array}{l}\text { DSN } \\
(98.7\end{array}$ \\
\hline & Shinella & CCNWSP92 & - & - & - & $\begin{array}{l}45.33 \pm \\
11.71\end{array}$ & ACCC19821 & KF735807 & $\begin{array}{l}\text { Shin } \\
\text { zoos } \\
196:\end{array}$ \\
\hline & Stenotrophomonas & CCNWSP15 & - & $2.22 \pm 0.11$ & $2.38 \pm 0.22$ & $\begin{array}{l}56.20 \pm \\
6.03\end{array}$ & ACCC19830 & KF735811 & $\begin{array}{l}\text { Sten } \\
\text { malt } \\
434^{7}\end{array}$ \\
\hline
\end{tabular}

a The ratio of the halo diameter to the colony diameter was used to indicate the ability of an isolate to solubilize mineral phosphate and produce siderophore Data are presented as means \pm standard errors $(n=3)$.

b - Negative for the trait.

Among 12 of the 13 rhizobacterial genera (all excluding the rhizobial genus Ensifer), 21 isolates were selected for the subsequent experiments (Table 2). In the single inoculation experiment without the rhizobium, seven isolates, namely CCNWSP46, CCNWSP13-4, CCNWSP78, CCNWSP92, CCNWSP15, CCNWSP60, and CCNWSP26, significantly promoted plant growth in terms of increasing root dry weight (by $38-62 \%$ ) and shoot dry weight (by $34-51 \%$ ). None of these 21 isolates formed nodules with soybean roots (Table 3; detailed data are shown in Online Resource 1). 
Table 3

The Ratio of the agronomic parameters of the inoculated soybean plants to the non-inoculated plants

\begin{tabular}{|c|c|c|c|}
\hline \multirow[t]{2}{*}{ Treatment } & \multicolumn{2}{|l|}{ Dry weight ${ }^{a}$} & \multirow[t]{2}{*}{ Total number of root nodules } \\
\hline & Root & Shoot & \\
\hline No inoculation & $1.000 \pm 0.221$ & $1.000 \pm 0.205$ & 0 \\
\hline Inoculation with CCNWSP46 & $1.618 \pm 0.317^{\star \star *}$ & $1.379 \pm 0.317 \star \star$ & 0 \\
\hline Inoculation with CCNWSP13-4 & $1.502 \pm 0.295^{\star \star}$ & $1.506 \pm 0.197 \star \star$ & 0 \\
\hline Inoculation with CCNWSP78 & $1.462 \pm 0.185^{\star \star}$ & $1.344 \pm 0.272^{\star}$ & 0 \\
\hline Inoculation with CCNWSP92 & $1.423 \pm 0.437^{\star}$ & $1.430 \pm 0.231^{\star \star}$ & 0 \\
\hline Inoculation with CCNWSP15 & $1.519 \pm 0.289 * *$ & $1.514 \pm 0.406^{\star \star}$ & 0 \\
\hline Inoculation with CCNWSP60 & $1.381 \pm 0.311^{\star}$ & $1.389 \pm 0.292^{\star \star}$ & 0 \\
\hline Inoculation with CCNWSP31 & $1.311 \pm 0.370$ & $1.308 \pm 0.233$ & 0 \\
\hline Inoculation with CCNWSP26 & $1.425 \pm 0.309 * *$ & $1.185 \pm 0.298$ & 0 \\
\hline Inoculation with CCNWSP76 & $1.261 \pm 0.247$ & $1.144 \pm 0.238$ & 0 \\
\hline Inoculation with CCNWSP2 & $1.104 \pm 0.244$ & $1.102 \pm 0.228$ & 0 \\
\hline Inoculation with CCNWSP68 & $1.267 \pm 0.447$ & $1.254 \pm 0.281$ & 0 \\
\hline Inoculation with CCNWSP10 & $0.882 \pm 0.227$ & $1.090 \pm 0.247$ & 0 \\
\hline Inoculation with CCNWSP11 & $1.279 \pm 0.299$ & $1.176 \pm 0.242$ & 0 \\
\hline Inoculation with CCNWSP27 & $1.015 \pm 0.123$ & $1.169 \pm 0.273$ & 0 \\
\hline Inoculation with CCNWSP13-2 & $1.086 \pm 0.346$ & $1.053 \pm 0.264$ & 0 \\
\hline Inoculation with CCNWSP30 & $1.169 \pm 0.292$ & $1.132 \pm 0.196$ & 0 \\
\hline Inoculation with CCNWSP21 & $1.231 \pm 0.278$ & $1.252 \pm 0.443$ & 0 \\
\hline Inoculation with CCNWSP4 & $1.077 \pm 0.202$ & $1.154 \pm 0.257$ & 0 \\
\hline Inoculation with CCNWSP33 & $0.975 \pm 0.316$ & $1.010 \pm 0.320$ & 0 \\
\hline Inoculation with CCNWSP21-1 & $1.178 \pm 0.341$ & $1.162 \pm 0.250$ & 0 \\
\hline Inoculation with CCNWSP25 & $1.117 \pm 0.507$ & $1.012 \pm 0.273$ & 0 \\
\hline
\end{tabular}

All of the 21 rhizobacterial isolates were screened for their ability to metabolize 71 nutrient substrates (Fig. 1; detailed data are shown in Online Resource 2). It was found that these metabolic phenotypes were genus specific. The isolates belonging to the same genus had similar metabolic phenotypes with respect to the utilization of various nutrient substrates. Meanwhile, the isolates of different genera showed diverse metabolic patterns. Some of the isolates (CCNWSP92, CCNWSP15, CCNWSP60, and CCNWSP26), which exhibited better PGP effects in the single inoculation experiment, showed stronger abilities to utilize multiple sugar substrates, such as D-Maltose, D-trehalose, D-cellobiose, and gentiobiose. However, other isolates (CCNWSP13-4 and CCNWSP78) showed a different pattern of nutrient substrate metabolism; they could not use those more available sugar substrates, but were able to utilize $p$-hydroxyphenylacetic acid, which is a carbon source that is harder to use.

Effects of rhizobacterial isolates on the soybean-rhizobium symbiosis system

The 21 rhizobacterial isolates were tested in the co-inoculation experiments. Of these, 13 isolates showed capacity to markedly increase at least one plant agronomic parameter (Table 4; detailed data are shown in Online Resource 3 ). When co-inoculated with the rhizobium CCNWSX1528, 10 isolates significantly increased the RDW by $19-63 \%, 7$ isolates increased the SDW by $16-30 \%, 6$ isolates increased the NDW by $22-40 \%, 8$ isolates increased the TNN by $21-66 \%$, 8 isolates increased the RNN by $30-97 \%, 10$ isolates increased the RN by $19-56 \%$, and 6 isolates increased the SN by $17-26 \%$. These 13 isolates increased the $\mathrm{Cl}$ by $5-51 \%$. 
Table 4

The Ratio of the agronomic parameters of soybean plants co-inoculated with rhizobacteria and Sinorhizobium sp. CCNWSX1528 to those inoculated with Sinorhizobium sp. CCNWSX1528 alone

\begin{tabular}{|c|c|c|c|c|c|c|c|c|}
\hline \multirow[t]{2}{*}{ Treatment } & \multicolumn{3}{|c|}{ Dry weight } & \multicolumn{2}{|c|}{ Number of nodules } & \multicolumn{2}{|c|}{ Total nitrogen } & \multirow{2}{*}{$\begin{array}{l}\text { Comprehensive } \\
\text { index }\end{array}$} \\
\hline & Root & Shoot & Per nodule & $\begin{array}{l}\text { Total } \\
\text { nodules }\end{array}$ & $\begin{array}{l}\text { Red } \\
\text { nodules }\end{array}$ & Root & Shoot & \\
\hline CCNWSX1528 alone & $\begin{array}{l}1.000 \pm \\
0.081\end{array}$ & $\begin{array}{l}1.000 \pm \\
0.088\end{array}$ & $\begin{array}{l}1.000 \pm \\
0.147\end{array}$ & $\begin{array}{l}1.000 \pm \\
0.077\end{array}$ & $\begin{array}{l}1.000 \pm \\
0.154\end{array}$ & $\begin{array}{l}1.000 \pm \\
0.080\end{array}$ & $\begin{array}{l}1.000 \pm \\
0.124\end{array}$ & 1.000 \\
\hline $\begin{array}{l}\text { CCNWSX1528 + } \\
\text { CCNWSP46 }\end{array}$ & $\begin{array}{l}1.631 \pm \\
0.243^{\star \star}\end{array}$ & $\begin{array}{l}1.295 \pm \\
0.153^{\star \star}\end{array}$ & $\begin{array}{l}1.397 \pm \\
0.446^{\star \star}\end{array}$ & $\begin{array}{l}1.582 \pm \\
0.164^{\star \star}\end{array}$ & $\begin{array}{l}1.922 \pm \\
0.237^{\star \star}\end{array}$ & $\begin{array}{l}1.560 \pm \\
0.205^{\star \star}\end{array}$ & $\begin{array}{l}1.212 \pm \\
0.104^{\star \star}\end{array}$ & 1.514 \\
\hline $\begin{array}{l}\text { CCNWSX1528 + } \\
\text { CCNWSP13-4 }\end{array}$ & $\begin{array}{l}1.451 \pm \\
0.125^{\star \star}\end{array}$ & $\begin{array}{l}1.191 \pm \\
0.076^{\star \star}\end{array}$ & $\begin{array}{l}1.222 \pm \\
0.126^{\star}\end{array}$ & $\begin{array}{l}1.661 \pm \\
0.139 \star \star\end{array}$ & $\begin{array}{l}1.970 \pm \\
0.470^{\star \star}\end{array}$ & $\begin{array}{l}1.369 \pm \\
0.140^{\star \star}\end{array}$ & 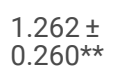 & 1.447 \\
\hline $\begin{array}{l}\text { CCNWSX1528 + } \\
\text { CCNWSP78 }\end{array}$ & $\begin{array}{l}1.513 \pm \\
0.206^{\star \star}\end{array}$ & $\begin{array}{l}1.123 \pm \\
0.088\end{array}$ & $\begin{array}{l}1.182 \pm \\
0.196\end{array}$ & $\begin{array}{l}1.496 \pm \\
0.200 \star \star\end{array}$ & $\begin{array}{l}1.385 \pm \\
0.302^{\star \star}\end{array}$ & $\begin{array}{l}1.401 \pm \\
0.248^{\star \star}\end{array}$ & $\begin{array}{l}1.173 \pm \\
0.118^{\star}\end{array}$ & 1.325 \\
\hline $\begin{array}{l}\text { CCNWSX1528 + } \\
\text { CCNWSP92 }\end{array}$ & $\begin{array}{l}1.327 \pm \\
0.240^{\star \star}\end{array}$ & $\begin{array}{l}1.182 \pm \\
0.123^{\star \star}\end{array}$ & $\begin{array}{l}1.134 \pm \\
0.119\end{array}$ & $\begin{array}{l}1.444 \pm \\
0.152^{\star \star}\end{array}$ & $\begin{array}{l}1.704 \pm \\
0.194^{\star \star}\end{array}$ & $\begin{array}{l}1.248 \pm \\
0.205^{\star \star}\end{array}$ & $\begin{array}{l}1.168 \pm \\
0.165^{\star}\end{array}$ & 1.315 \\
\hline $\begin{array}{l}\text { CCNWSX1528 + } \\
\text { CCNWSP15 }\end{array}$ & $\begin{array}{l}1.386 \pm \\
0.191^{\star \star}\end{array}$ & $\begin{array}{l}1.155 \pm \\
0.156^{\star \star}\end{array}$ & $\begin{array}{l}1.128 \pm \\
0.114\end{array}$ & $\begin{array}{l}1.353 \pm \\
0.147^{\star \star}\end{array}$ & $\begin{array}{l}1.553 \pm \\
0.218^{\star \star}\end{array}$ & $\begin{array}{l}1.387 \pm \\
0.208^{\star \star}\end{array}$ & $\begin{array}{l}1.224 \pm \\
0.132^{\star \star}\end{array}$ & 1.312 \\
\hline $\begin{array}{l}\text { CCNWSX1528 + } \\
\text { CCNWSP60 }\end{array}$ & $\begin{array}{l}1.333 \pm \\
0.116^{\star \star}\end{array}$ & $\begin{array}{l}1.201 \pm \\
0.061^{\star \star}\end{array}$ & $\begin{array}{l}1.248 \pm \\
0.239 *\end{array}$ & $\begin{array}{l}1.241 \pm \\
0.136^{\star \star}\end{array}$ & $\begin{array}{l}1.350 \pm \\
0.240^{\star \star}\end{array}$ & $\begin{array}{l}1.314 \pm \\
0.146^{\star \star}\end{array}$ & $\begin{array}{l}1.154 \pm \\
0.059\end{array}$ & 1.263 \\
\hline $\begin{array}{l}\text { CCNWSX1528 + } \\
\text { CCNWSP31 }\end{array}$ & $\begin{array}{l}1.232 \pm \\
0.216^{\star \star}\end{array}$ & $\begin{array}{l}1.202 \pm \\
0.138^{\star \star}\end{array}$ & $\begin{array}{l}1.326 \pm \\
0.099 * \star\end{array}$ & $\begin{array}{l}1.206 \pm \\
0.132^{\star}\end{array}$ & $\begin{array}{l}1.333 \pm \\
0.183^{\star \star}\end{array}$ & $\begin{array}{l}1.278 \pm \\
0.145^{\star \star}\end{array}$ & $\begin{array}{l}1.116 \pm \\
0.133\end{array}$ & 1.242 \\
\hline $\begin{array}{l}\text { CCNWSX1528 + } \\
\text { CCNWSP26 }\end{array}$ & $\begin{array}{l}1.313 \pm \\
0.159 * \star\end{array}$ & $\begin{array}{l}1.215 \pm \\
0.141^{\star \star}\end{array}$ & $\begin{array}{l}1.313 \pm \\
0.135^{\star \star}\end{array}$ & $\begin{array}{l}1.158 \pm \\
0.104\end{array}$ & $\begin{array}{l}1.184 \pm \\
0.116\end{array}$ & $\begin{array}{l}1.323 \pm \\
0.137^{\star \star}\end{array}$ & $\begin{array}{l}1.150 \pm \\
0.188\end{array}$ & 1.237 \\
\hline $\begin{array}{l}\text { CCNWSX1528 + } \\
\text { CCNWSP76 }\end{array}$ & $\begin{array}{l}1.199 \pm \\
0.077^{*}\end{array}$ & $\begin{array}{l}1.059 \pm \\
0.071\end{array}$ & $\begin{array}{l}1.019 \pm \\
0.118\end{array}$ & $\begin{array}{l}1.336 \pm \\
0.127^{\star \star}\end{array}$ & $\begin{array}{l}1.225 \pm \\
0.176\end{array}$ & $\begin{array}{l}1.185 \pm \\
0.074^{\star}\end{array}$ & $\begin{array}{l}1.098 \pm \\
0.143\end{array}$ & 1.160 \\
\hline $\begin{array}{l}\text { CCNWSX1528 + } \\
\text { CCNWSP2 }\end{array}$ & $\begin{array}{l}1.046 \pm \\
0.125\end{array}$ & $\begin{array}{l}1.094 \pm \\
0.124\end{array}$ & $\begin{array}{l}1.335 \pm \\
0.189 \star \star\end{array}$ & $\begin{array}{l}1.068 \pm \\
0.121\end{array}$ & $\begin{array}{l}1.169 \pm \\
0.183\end{array}$ & $\begin{array}{l}1.103 \pm \\
0.132\end{array}$ & $\begin{array}{l}1.085 \pm \\
0.126\end{array}$ & 1.129 \\
\hline $\begin{array}{l}\text { CCNWSX1528 + } \\
\text { CCNWSP68 }\end{array}$ & $\begin{array}{l}1.167 \pm \\
0.235\end{array}$ & $\begin{array}{l}1.044 \pm \\
0.101\end{array}$ & $\begin{array}{l}1.094 \pm \\
0.180\end{array}$ & $\begin{array}{l}1.086 \pm \\
0.258\end{array}$ & $\begin{array}{l}1.104 \pm \\
0.154\end{array}$ & $\begin{array}{l}1.192 \pm \\
0.270 *\end{array}$ & $\begin{array}{l}1.200 \pm \\
0.158^{\star \star}\end{array}$ & 1.127 \\
\hline $\begin{array}{l}\text { CCNWSX1528 + } \\
\text { CCNWSP10 }\end{array}$ & $\begin{array}{l}1.193 \pm \\
0.136^{*}\end{array}$ & $\begin{array}{l}1.038 \pm \\
0.051\end{array}$ & $\begin{array}{l}1.136 \pm \\
0.198\end{array}$ & $\begin{array}{l}1.095 \pm \\
0.209\end{array}$ & $\begin{array}{l}1.226 \pm \\
0.295\end{array}$ & $\begin{array}{l}1.125 \pm \\
0.129\end{array}$ & $\begin{array}{l}0.994 \pm \\
0.106\end{array}$ & 1.115 \\
\hline $\begin{array}{l}\text { CCNWSX1528 + } \\
\text { CCNWSP11 }\end{array}$ & $\begin{array}{l}1.078 \pm \\
0.117\end{array}$ & $\begin{array}{l}1.012 \pm \\
0.096\end{array}$ & $\begin{array}{l}1.084 \pm \\
0.171\end{array}$ & $\begin{array}{l}1.108 \pm \\
0.156\end{array}$ & $\begin{array}{l}1.280 \pm \\
0.248\end{array}$ & $\begin{array}{l}1.096 \pm \\
0.126\end{array}$ & $\begin{array}{l}1.096 \pm \\
0.122\end{array}$ & 1.108 \\
\hline $\begin{array}{l}\text { CCNWSX1528 + } \\
\text { CCNWSP27 }\end{array}$ & $\begin{array}{l}0.937 \pm \\
0.110\end{array}$ & $\begin{array}{l}1.012 \pm \\
0.055\end{array}$ & $\begin{array}{l}1.019 \pm \\
0.106\end{array}$ & $\begin{array}{l}1.120 \pm \\
0.151\end{array}$ & $\begin{array}{l}1.295 \pm \\
0.187^{\star}\end{array}$ & $\begin{array}{l}0.881 \pm \\
0.142\end{array}$ & $\begin{array}{l}1.062 \pm \\
0.063\end{array}$ & 1.047 \\
\hline $\begin{array}{l}\text { CCNWSX1528 + } \\
\text { CCNWSP13-2 }\end{array}$ & $\begin{array}{l}1.010 \pm \\
0.111\end{array}$ & $\begin{array}{l}0.936 \pm \\
0.109\end{array}$ & $\begin{array}{l}0.909 \pm \\
0.118\end{array}$ & $\begin{array}{l}1.051 \pm \\
0.208\end{array}$ & $\begin{array}{l}1.202 \pm \\
0.314\end{array}$ & $\begin{array}{l}1.008 \pm \\
0.104\end{array}$ & $\begin{array}{l}0.999 \pm \\
0.140\end{array}$ & 1.016 \\
\hline $\begin{array}{l}\text { CCNWSX1528 + } \\
\text { CCNWSP30 }\end{array}$ & $\begin{array}{l}1.060 \pm \\
0.199\end{array}$ & $\begin{array}{l}0.972 \pm \\
0.097\end{array}$ & $\begin{array}{l}0.986 \pm \\
0.171\end{array}$ & $\begin{array}{l}1.020 \pm \\
0.086\end{array}$ & $\begin{array}{l}1.063 \pm \\
0.179\end{array}$ & $\begin{array}{l}1.008 \pm \\
0.160\end{array}$ & $\begin{array}{l}0.974 \pm \\
0.094\end{array}$ & 1.012 \\
\hline $\begin{array}{l}\text { CCNWSX1528 + } \\
\text { CCNWSP21 }\end{array}$ & $\begin{array}{l}1.093 \pm \\
0.143\end{array}$ & $\begin{array}{l}1.005 \pm \\
0.183\end{array}$ & $\begin{array}{l}1.209 \pm \\
0.204\end{array}$ & $\begin{array}{l}0.770 \pm \\
0.170 *\end{array}$ & $\begin{array}{l}0.890 \pm \\
0.135\end{array}$ & $\begin{array}{l}1.109 \pm \\
0.095\end{array}$ & $\begin{array}{l}1.007 \pm \\
0.150\end{array}$ & 1.012 \\
\hline $\begin{array}{l}\text { CCNWSX1528 + } \\
\text { CCNWSP4 }\end{array}$ & $\begin{array}{l}1.000 \pm \\
0.118\end{array}$ & $\begin{array}{l}0.977 \pm \\
0.055\end{array}$ & $\begin{array}{l}1.134 \pm \\
0.143\end{array}$ & $\begin{array}{l}0.973 \pm \\
0.086\end{array}$ & $\begin{array}{l}1.082 \pm \\
0.208\end{array}$ & $\begin{array}{l}0.933 \pm \\
0.092\end{array}$ & $\begin{array}{l}0.958 \pm \\
0.145\end{array}$ & 1.008 \\
\hline $\begin{array}{l}\text { CCNWSX1528 + } \\
\text { CCNWSP33 }\end{array}$ & $\begin{array}{l}1.142 \pm \\
0.162\end{array}$ & $\begin{array}{l}0.911 \pm \\
0.140\end{array}$ & $\begin{array}{l}0.908 \pm \\
0.229\end{array}$ & $\begin{array}{l}0.878 \pm \\
0.194\end{array}$ & $\begin{array}{l}0.872 \pm \\
0.327\end{array}$ & $\begin{array}{l}1.094 \pm \\
0.104\end{array}$ & $\begin{array}{l}0.999 \pm \\
0.150\end{array}$ & 0.972 \\
\hline $\begin{array}{l}\text { CCNWSX1528 + } \\
\text { CCNWSP21-1 }\end{array}$ & $\begin{array}{l}0.984 \pm \\
0.126\end{array}$ & $\begin{array}{l}0.939 \pm \\
0.083\end{array}$ & $\begin{array}{l}0.907 \pm \\
0.187\end{array}$ & $\begin{array}{l}0.924 \pm \\
0.181\end{array}$ & $\begin{array}{l}0.950 \pm \\
0.119\end{array}$ & $\begin{array}{l}0.994 \pm \\
0.088\end{array}$ & $\begin{array}{l}0.966 \pm \\
0.118\end{array}$ & 0.952 \\
\hline $\begin{array}{l}\text { CCNWSX1528 + } \\
\text { CCNWSP25 }\end{array}$ & $\begin{array}{l}0.917 \pm \\
0.225\end{array}$ & $\begin{array}{l}0.869 \pm \\
0.158\end{array}$ & $\begin{array}{l}1.016 \pm \\
0.206\end{array}$ & $\begin{array}{l}0.822 \pm \\
0.377\end{array}$ & $\begin{array}{l}0.990 \pm \\
0.389\end{array}$ & $\begin{array}{l}1.002 \pm \\
0.176\end{array}$ & $\begin{array}{l}0.945 \pm \\
0.127\end{array}$ & 0.937 \\
\hline
\end{tabular}

The 13 isolates belonged to nine genera, namely Bacillus (4 isolates), Alcaligenes (2 isolates), Stenotrophomonas (1 isolate), Microbacterium (1 isolate), Sphingobacterium (1 isolate), Agrobacterium (1 isolate), Pseudochrobactrum (1 isolate), Brevundimonas (1 isolate), and Paracoccus (1 isolate). There were only three genera in which the isolates did not show significant PGP ability. Eight isolates (CCNWSP46, CCNWSP13-4, CCNWSP78, CCNWSP92, CCNWSP15, CCNWSP60, CCNWSP31, and CCNWSP26) showed better PGP effects with respect to almost all parameters and had the highest $\mathrm{Cl}$ values (1.237-1.514). 
Meanwhile, the $\mathrm{Cl}$ values of the other isolates were less than 1.2. Among the eight isolates with the best PGP effects, only CCNWSP31 did not significantly promote plant growth in the single inoculation experiment without rhizobium.

The correlations between rhizobacterial phenotypes and PGP ability

Spearman's correlation analysis was carried out to explore the potential relationships between the eight soybean plant parameters (RDW, SDW, NDW, TNN, $\mathrm{RNN}, \mathrm{RN}, \mathrm{SN}$, and $\mathrm{Cl}$ ) and the multiple phenotypes of the rhizobacterial isolates, including four PGP traits (solubilizing phosphate and producing IAA, chitinase, and siderophores) and 71 metabolic phenotypes (WCD, Biolog ${ }^{\text {TM }}$ GenIII). The improvement of plant parameters was significantly correlated with the metabolism of some substrates, but not with any PGP traits (Fig. 2; detailed data are shown in Online Resource 4).

The metabolism of 11 substrates was significantly positively related to PGP ability. Specifically, D-melibiose utilization was related to an increase in RDW ( $r=$ 0.443). D-Maltose, D-trehalose, D-cellobiose, sucrose, $a-D$-lactose, $\beta$-methyl-D-glucoside, D-salicin, and pectin utilization was related to an increase in SDW ( $r=$ $0.435-0.621)$. D-Cellobiose, $\beta$-methyl-D-glucoside, and D-salicin utilization was related to an increase in TNN $(r=0.443-0.521)$. Dextrin, D-cellobiose, $\beta$-methyl$\mathrm{D}$-glucoside, D-salicin, and $p$-hydroxyphenylacetic acid utilization was related to an increase in RNN $(r=0.435-0.530)$. D-Melibiose utilization was related to an increase RN ( $r=0.437)$. D-Maltose, $\beta$-methyl-D-glucoside, and D-salicin utilization was related to an increase in $\mathrm{Cl}(r=0.435-0.510)$. In addition, the metabolism of three substrates was significantly negatively related to PGP ability. That is, L-aspartic acid utilization was related to RNN ( $r=-0.453)$, a-ketoglutaric acid utilization was related to NDW $(r=-0.433)$, and formic acid utilization was related to RDW $(r=-0.475)$ and RN $(r=-0.499)$.

\section{Discussion}

Selection of test isolates, plant agronomic parameters, and PGP traits

This study was carried out to explore the possibility of using multiple metabolic phenotypes as criteria for the preliminary screening of PGPR. The aim of the study was achieved by evaluating the correlations between the metabolic phenotypes of PGPR isolates and their PGP ability, and between the PGP traits of the isolates and their PGP ability. In order to obtain more representative criteria, focus was placed on the selection of test isolates, plant agronomic parameters, and PGP traits when designing the experiment.

Among the 90 rhizobacterial isolates obtained in this study, Proteobacteria (51 isolates) was the most dominant phylum, followed by Firmicutes ( 33 isolates). In addition, Bacteroidetes (5 isolates) and Actinobacteria ( 1 isolate) were identified. There is a general assumption that fast-growing, easily cultivable Proteobacteria are the dominant rhizosphere colonizers (Kristen et al. 2009). Xu et al. (2009) reported that Proteobacteria, Actinobacteria, Bacteroidetes, Nitrospirae, Firmicutes, Verrucomicrobia, and Acidobacteria could colonize the soybean rhizosphere, with Proteobacteria being the dominant group ( $>40 \%$ of the bacterial community). Zhang et al. (2018) also found that Proteobacteria, Firmicutes, and Actinobacteria were the prominent phyla in the rhizosphere microbial communities of soybean at 50 sites across China. The results of the present study are thus consistent with these previous findings.

At the genus level, the dominant rhizobacteria isolated in the present study were Bacillus (24 isolates), Alcaligenes (17 isolates), Pseudochrobactrum (16 isolates), Lysinibacillus (9 isolates), and Agrobacterium (3 isolates). Besides Ensifer, which is a common symbiotic rhizobial genus, some of the other genera isolated in this study have also been previously identified as potential sources of PGPR (Khan et al. 2017; Rahmoune et al. 2017; Vessey 2003). Here, 21 isolates were selected from the 90 rhizobacterial isolates to represent the 12 genera (all except Ensifer) as follows: four isolates were selected from each of the three genera containing the largest number of isolates (Bacillus, Alcaligenes, and Pseudochrobactrum), and one isolate was selected from each of the remaining nine genera (Microbacterium, Sphingobacterium, Lysinibacillus, Advenella, Agrobacterium, Brevundimonas, Paracoccus, Shinella, and Stenotrophomonas). The co-inoculation results showed that PGPR isolates accounted for a large proportion of both the genera (9/12) and isolates (13/21). In accordance with the results of previous studies (Backer et al. 2018; Khan et al. 2017; Rahmoune et al. 2017; Vessey 2003), the rhizobacterial isolates in the present study that belonged to Bacillus, Alcaligenes, Stenotrophomonas, Microbacterium, Sphingobacterium, and Agrobacterium were associated with significant PGP effects. This indicates that there is a large number of PGPR inhabiting the soybean rhizosphere, and they are widely distributed across several different genera.

In this study, seven plant agronomic parameters (such as RDW, SDW, and NDW) and one index (Cl) were used to describe the PGP effects of rhizobacterial isolates. PGPR can affect plants through various and complex pathways (Backer et al. 2018; Rodriguez et al. 2019). The diversity of PGP mechanisms means that multiple plant agronomic parameters may change due to the action of PGPR. So, the standards for evaluating the performance of PGPR should include not only plant growth traits, such as shoot elongation, plant height, plant biomass, and yield (Tabassum et al. 2017), but also more targeted indicators, such as root architecture, flowering time, and plant enzyme activity, in some specific cases (Chaney and Baucomn 2020; Chu et al. 2020; Lee et al. 2020; Mahdavi et al. 2020). In addition, a variety of comprehensive indexes calculated based on several plant parameters have previously been used as the main standard to evaluate the quality of fruit, plant vigor, and physiological parameters. These comprehensive indexes are considered more representative than single parameters (Dey and Raghuwanshi 2020; Guo et al. 2019; Wang et al., 2011).

In the present study, a soybean-rhizobium symbiosis system was used to evaluate the PGP effects of rhizobacterial isolates on plants. Comparing the plant agronomic parameters under the two experimental conditions (with and without the rhizobium), it was found that seven isolates that showed PGP effects in the single inoculation experiment, could also promote the symbiosis in co-inoculation experiments. Interestingly, six isolates that did not show PGP effects in the single inoculation experiment, increased at least one plant agronomic parameter in co-inoculation experiments. This phenomenon suggests that, in addition to directly regulating plant growth in the soybean-rhizobium symbiosis system, the PGPR isolates could act via other PGP mechanisms, such as by facilitating symbiotic nodulation. Therefore, seven plant agronomic parameters, including two related to plant growth (RDW and SDW), three related to root nodulation (TNN, RNN, and NDW), and two related to nitrogen fixation (RN and SN), were selected and integrated into one index (CI) to assess the effects of PGPR on plant growth and symbiotic nodulation through multiple mechanisms.

Page $12 / 21$ 
Here, four traditional PGP traits were selected to evaluate their reliability as pre-screening criteria for PGPR in the present study. Two of the traits, mineral phosphorus solubilization and siderophore production, are associated with nutrient availability. Mineral phosphorus solubilization could increase soil phosphorus availability in the environment where available phosphorus is scarce and mineral phosphorus is abundant (Brito et al. 2020). Siderophore production could increase the absorption of iron by plants, or could control plant pathogens by depriving them of iron in an iron-deficient environment (Ghazy and El-Nahrawy 2020; Gopalakrishnan et al. 2015). In order to create conditions for these two traits to function, $\mathrm{Ca}_{3}\left(\mathrm{PO}_{4}\right)_{2}$ and $\mathrm{FeCl}_{3}$ were added to the sterile substrate as the main phosphorus and iron sources, respectively. The two further traits tested in this study were IAA and chitinase production. IAA is one of the most important auxins produced by PGPR and thus IAA production can be directly associated with plant growth (Spaepen and Vanderleyden 2011). Meanwhile, chitinase production may affect nodulation by hydrolyzing the nod factor, which is an important signaling molecule involved in nodulation (Jung et al. 2008).

Many studies have used these four PGP traits described above as the main markers of PGPR (Li et al. 2020; Toscano-Verduzco et al. 2020). However, in the present study, no significant correlations were found between these four PGP traits and the actual PGP abilities of the rhizobacterial isolates. Moreover, one isolate (CCNWSP10), which did not display any of these four PGP traits, showed PGP effects in the inoculation experiments. The reliability of using PGP traits as the primary basis for the preliminary screening of PGPR in the laboratory has also been questioned by other researchers. These questions have arisen because the action of these PGP mechanisms is dependent on the environment and there are many other unknown PGP mechanisms (Backer et al. 2018; Finkel et al. 2017). Therefore, instead of the traditional PGP traits, other traits that have stronger correlations with the actual PGP abilities should be identified and used as indicators for primary PGPR screening.

Feasibility of using metabolic phenotypes as screening indicators

In contrast to the PGP traits, the metabolic phenotypes of microorganisms, with respect to the utilization of nutrient substrates found in root exudates, are closely related to the ability of the microorganisms to colonize the rhizosphere of plants. This colonization is a necessary prerequisite for plant-microbe interactions (Hassan et al. 2019; Zhalnina et al. 2018). It can be proposed that the capacity of PGPR to utilize nutrient substrates may be a more universal and stable predictor of their possible effects on plants. Therefore, in the present study, rhizobacterial isolates were screened for their ability to utilize multiple nutrient substrates. It was found that 11 of the nutrient utilization phenotypes (including dextrin, D-maltose, D-trehalose, and D-cellobiose) were significantly positively correlated with increases in specific plant agronomic traits. These nutrient substrates are common components of root exudates, and some PGPR strains have previously been shown to be able to metabolize them (Prasanna et al. 2010; Zhang et al. 2020).

In the present study, D-salicin, $\beta$-methyl-D-glucoside, and D-cellobiose utilization were found to be the only three phenotypes that were most strongly correlated with three plant agronomic parameters (SDW, TNN, and RNN). Further, the phenotypes of D-salicin and $\beta$-methyl-D-glucoside utilization were also related to CI. Salicin is an aryl $\beta$-glucoside that is found in a wide variety of plants and is used as a carbon source by many bacteria, including potential PGPR and phytopathogenic bacteria (Charaoui-Boukerzaza and Hugouvieux-Cotte-Pattat 2013). In addition, salicin can be metabolized to salicylic acid (Mahdi 2014), which is a plant immune system-related signaling molecule that plays a role in plant-microbe interactions and root microbiome regulation (Conrath et al. 2002; Lebeis et al. 2015). $\beta$-Methyl-D-glucoside and cellobiose can act as key carbon sources that induce changes in the rhizosphere microbial community composition (Dong et al. 2016; Shen et al. 2020). The results of the present study further confirm the importance of these three substrates for PGPR.

Of the remaining nutrient substrates, D-melibiose utilization was a phenotype that was found to be correlated with two agronomic parameters (RDW and RN). Melibiose is widely present in plant seeds; it can be released into the rhizosphere and thereby utilized by PGPR isolates (Andersen et al. 2005; Bringhurst et al. 2001). The assimilation of melibiose is crucial for plant pathogens to gain an advantage in the rhizosphere (Meyer et al. 2018). In addition, D-trehalose and dextrin utilization showed relatively strong correlations ( $r>0.5$ ) with plant growth (SDW) and nodulation (RNN), respectively. Trehalose can act as a signaling metabolite in symbiotic nodulation or immune responses (Lunn et al. 2014; Wang et al. 2020), and it has been shown to help maintain the activity of PGPR (Gopi et al. 2019; Radha and Rao 2014). Dextrin is also an available carbon source for PGPR that has been reported on before (Silini-Cherif et al. 2012). Furthermore, maltose, sucrose, lactose, and pectin are all common components in root exudates and have been shown, in many studies, to be related to the colonization and activity of PGPR (Al-Ali et al. 2018; Chudzik et al. 2018; Li et al. 2012; Mise et al. 2020; Patel et al. 2008; Wu et al. 2015). In these previous studies, it has been suggested that the utilization of these root exudate-related nutrient substrates may be meaningful for PGPR. The results of the present study supported this view and further suggested that the capacities of PGPR isolates to utilize these nutrient substances could be key to their effects on plant growth and symbiotic nodulation. Thus, the utilization of these substrates could be used as an indicator for the pre-screening of PGPR isolates.

There are some problems that must be considered when using these metabolic phenotypes as screening indicators. First, the isolates of different taxa might have preferences for various types of nutrient substrates in root exudates when colonizing. Therefore, to ensure that isolates of different taxa are included in the evaluation, a sufficient variety of nutrient substrates should be selected when designing the screening indicator system. For example, in the present study, the eight top PGPR isolates (with respect to PGP ability and $\mathrm{Cl}$ value) showed different abilities to utilize the 11 substrates that were positively associated with PGP ability. In particular, the two A/caligenes isolates, CCNWSP13-4 and CCNWSP78, displayed poor abilities to metabolize most of these 11 substrates, but they could utilize $p$-hydroxyphenylacetic acid. Some Alcaligenes strains have been shown to be capable of degrading polycyclic aromatic hydrocarbons (Sun et al. 2017). Kragelund and Nybroe (1996) reported that 2,4-dichlorophenoxyacetic acid was the main carbon source for an Alcaligenes strain during rhizosphere colonization. Therefore, for the isolates of certain taxa, such as Alcaligenes, their preferred nutrient substrates must be considered in the development of screening criteria.

A second issue with using metabolic phenotypes as indicators is that the metabolism of nutrients by an isolate may be only a prerequisite for its action on plants; it does not necessarily mean that the isolate can promote plant growth. In the present study, the four Alcaligenes isolates, CCNWSP13-2, CCNWSP13-4, CCNWSP30, and CCNWSP78, displayed similar metabolic phenotypes. However, only two of them (CCNWSP13-4 and CCNWSP78) could markedly promote 
plant growth. This might be because, in the case of effective colonization, these isolates have different PGP mechanisms that were not screened for in this study. Therefore, these metabolic phenotypes could be used as "necessary but not sufficient" criteria in the preliminary screening of PGPR.

In the present study, significant negative correlations were observed between the utilization of one amino acid and two organic acid substrates by the rhizobacterial isolates, and their PGP ability. Interestingly, in addition to these three substrates, the capacities of the isolates to utilize many other amino acid and organic acid substrates were also negatively correlated with their PGP ability, albeit not significantly. With regard to non-pathogenic microorganisms that colonize the root or rhizosphere, most studies have mainly focused on their positive effects on plant growth and related mechanisms. Little attention has been paid to the potential negative effects of these non-pathogenic microorganisms on plants (Backer et al. 2018; Vessey 2003). The results of the present study implied that some metabolic phenotypes of rhizobacteria may have a negative effect on plant growth. For example, low-molecular-weight organic acids are important components of root exudates and play effective roles in the dissolution of insoluble nutrients in the rhizosphere (Basak 2019). The utilization of low-molecular-weight organic acids by rhizobacteria may change the environment of the rhizosphere niche and reduce the availability of some nutrients (Fujii et al. 2013) such as tricalcium phosphate, which is the main phosphorus source for the plants used in this study.

Here, the correlations between the multiple metabolic phenotypes and PGP abilities of rhizobacterial isolates were demonstrated using sterilized vermiculite. Therefore, the results might not accurately represent the real relationships between the metabolic characteristics of rhizobacteria and their PGP abilities in actual farmland soil affected by physical, chemical, and biological factors. Thus, the results of this study might not be suitable for use as a direct measure of the practical PGP ability of rhizobacterial isolates in certain agricultural soil environments. However, these metabolic indicators identified in the artificially controlled environment are still of significance for the preliminary screening of PGPR. This is because these metabolic indictors simply describe the essential plant-microbe interactions with less external influence. The metabolic indicators may be more widely used in a variety of soils with different environmental characteristics than the criteria obtained using a specific soil condition. Using the metabolic indicators in this way will help to retain more rhizobacterial isolates that have unknown PGP mechanisms. This is essential for the goal of establishing a high-throughput screening system for PGPR.

\section{Conclusions}

Compared to some traditional PGP traits, metabolic phenotypes based on the ability of rhizobacterial isolates to metabolize nutrient substrates, show greater correlations with the PGP abilities of the isolates in the soybean-rhizobium symbiosis system. Based on this finding, it can be proposed that during preliminary PGPR screening, more attention should be paid to such metabolic phenotypes. These metabolic phenotypes may be related to the rhizosphere colonization ability of the isolates; colonization is the most important prerequisite for PGPR to affect plants. There are still many problems that need be resolved before applying these metabolic phenotypes as preliminary screening criteria for soybean PGPR. However, these phenotypes have great potential to become important indicators for preliminary PGPR screening. More metabolic phenotypes should be explored and evaluated based on a variety of plantmicrobe interaction systems to obtain sufficient screening criteria for PGPR strains of many different taxa. Through a combination of using these metabolic criteria and more PGP traits, it can be expected that a more efficient and versatile high-throughput screening system will be established for PGPR in the future.

\section{Abbreviations}

PGPR, plant growth-promoting rhizobacteria; IAA, indole-3-acetic acid; dpi, days post-inoculation; WCD, well color development; Cl, comprehensive index; RDW, root dry weight; SDW, shoot dry weight; NDW, dry weight of per nodule; TNN, total number of nodules; RNN, number of red nodules; RN, root total nitrogen; SN, shoot total nitrogen.

\section{Declarations}

Acknowledgements This work was supported by the National Natural Science Foundation of China $(41830755,31672241,31000943)$, the National Key Research \& Development Program of China (2018YFD0200404, 2016YFD0200308) and Key R\&D program of Shaanxi Province (2020ZDLNY07-09).

Availability of data The nucleotide sequence data reported are available in the NCBI GenBank databases under the accessions number (KF735789KF735811). The isolates used in this study are stored in Agricultural Culture Collection of China (ACCC).

Author contributions PS and GW designed the experiments with equal contribution. PS and JZ performed most of the experiments, including isolation and phenotypes scanning experiments. JZ, HL and LW performed the three inoculation experiments separately. XL, LZ, YZ and MC analyzed the data. PS and JZ wrote the article with equal contribution. All authors read and approved the final manuscript.

\section{References}

Al-Ali A, Deravel J, Krier F, Bechet M, Ongena M, Jacques P (2018) Biofilm formation is determinant in tomato rhizosphere colonization by Bacillus velezensis FZB42. Environ Sci Pollut Res 25: 29910-29920. https://doi.org/10.1007/s11356-017-0469-1

Ambrosini A, Beneduzi A, Stefanski T, Pinheiro FG, Vargas LK, Passaglia LMP (2012) Screening of plant growth promoting Rhizobacteria isolated from sunflower (Helianthus annuus L.). Plant Soil 356: 245-264. https://doi.org/10.1007/s11104-011-1079-1

Andersen KE, Bjergegaard C, Moller P, Sorensen JC, Sorensen H (2005) Compositional variations for alpha-galactosides in different species of Leguminosae, Brassicaceae, and barley: a chemotaxonomic study based on chemometrics and high-performance capillary electrophoresis. J Agric Food Chem 53: 5809-

5817. https://doi.org/10.1021/jf040471v 
Backer R, Rokem JS, I langumaran G, Lamont J, Praslickova D, Ricci E, Subramanian S, Smith DL (2018) Plant growth-promoting rhizobacteria: context, mechanisms of action, and roadmap to commercialization of biostimulants for sustainable agriculture. Front Plant Sci 9: 1473.

https://doi.org/10.3389/fpls.2018.01473

Badri DV, Vivanco JM (2009) Regulation and function of root exudates. Plant Cell Environ 32: 666-681. https://doi.org/10.1111/j.1365-3040.2009.01926.x

Bai Y, Muller DB, Srinivas G, Garrido-Oter R, Potthoff E, Rott M, Dombrowski N, Munch PC, Spaepen S, Remus-Emsermann M, Huttel B, McHardy AC, Vorholt JA, Schulze-Lefert P (2015) Functional overlap of the Arabidopsis leaf and root microbiota. Nature 528: 364-+. https://doi.org/10.1038/nature16192

Bais HP, Weir TL, Perry LG, Gilroy S, Vivanco JM (2006) The role of root exudates in rhizosphere interations with plants and other organisms. Annu Rev Plant Biol 57: 233-266. https://doi.org/10.1146/annurev.arplant.57.032905.105159

Basak BB (2019) Phosphorus release by low molecular weight organic acids from low-grade Indian rock phosphate. Waste Biomass Valori 10: $3225-3233$. https://doi.org/10.1007/s12649-018-0361-3

Bringhurst RM, Cardon ZG, Gage DJ (2001) Galactosides in the rhizosphere: utilization by Sinorhizobium meliloti and development of a biosensor. Proc Natl Acad Sci U S A 98: 4540-4545. https://doi.org/10.1073/pnas.071375898

Brito LF, Lopez MG, Straube L, Passaglia LMP, Wendischl VF (2020) Inorganic phosphate solubilization by rhizosphere bacterium Paenibacillus sonchi: gene expression and physiological functions. Front Microbiol 11: 588605. https://doi.org/10.3389/fmicb.2020.588605

Canto CD, Simonin M, King E, Moulin L, Bennett MJ, Castrillo G, Laplaze L (2020) An extended root phenotype: the rhizosphere, its formation and impacts on plant fitness. Plant J 103: 951-964. https://doi.org/10.1111/tpj.14781

Cattelan AJ, Hartel PG, Fuhrmann JJ (1999) Screening for plant growth-promoting rhizobacteria to promote early soybean growth. Soil Sci Soc Am J 63: 16701680. https://doi.org/10.2136/sssaj1999.6361670x

Chaney L, Baucomn RS (2020) The soil microbial community alters patterns of selection on flowering time and fitness-related traits in Ipomoea purpurea. Am J Bot 107: 186-194. https://doi.org/10.1002/ajb2.1426

Charaoui-Boukerzaza S, Hugouvieux-Cotte-Pattat N (2013) A family 3 glycosyl hydrolase of Dickeya dadantii 3937 is involved in the cleavage of aromatic glucosides. Microbiol-SGM 159: 2395-2404. https://doi.org/10.1099/mic.0.071407-0

Chu TN, Bui LV, Hoang MTT (2020) Pseudomonas PS01 isolated from maize rhizosphere alters root system architecture and promotes plant growth. Microorganisms 8: 471. https://doi.org/10.3390/microorganisms8040471

Chudzik B, Szczuka E, Leszczuk A, Strubinska J (2018) Modification of pectin distribution in sunflower (Helianthus annuus L.) roots in response to lead exposure. Environ Exp Bot 155: 251-259. https://doi.org/10.1016/j.envexpbot.2018.07.005

Conrath U, Pieterse CMJ, Mauch-Mani B (2002) Priming in plant-pathogen interactions. Trends Plant Sci 7: 210-216. https://doi.org/10.1016/s13601385(02)02244-6

Di Benedetto NA, Campaniello D, Bevilacqua A, Cataldi MP, Sinigaglia M, Flagella Z, Corbo MR (2019) Isolation, screening, and characterization of plantgrowth-promoting bacteria from durum wheat rhizosphere to improve $\mathrm{N}$ and $\mathrm{P}$ nutrient use efficiency. Microorganisms 7: 541.

https://doi.org/10.3390/microorganisms7110541

Dong Y, Dong K, Yang Z-x, Tang L, Zheng Y (2016) Rhizosphere microbial impacts of alleviating faba bean Fusariumwilt with inoculating AM fungi. Yingyong Shengtai Xuebao 27: 4029-4038. https://doi.org/10.13287/j.1001-9332.201612.022

Dey R, Raghuwanshi R (2020) Comprehensive assessment of growth parameters for screening endophytic bacterial strains in Solanum lycopersicum (Tomato). Heliyon 6: e05325. https://doi.org/10.1016/j.heliyon.2020.e05325

Egamberdieva D, Berg G, Lindstrom K, Rasanen LA (2013) Alleviation of salt stress of symbiotic Galega officinalis L. (goat's rue) by co-inoculation of Rhizobium with root-colonizing Pseudomonas. Plant Soil 369: 453-465. https://doi.org/10.1007/s11104-013-1586-3

Faure D, Desair J, Keijers V, Ali Bekri M, Proost P, Henrissat B, Vanderleyden J (1999) Growth of Azospirillum irakense KBC1 on the Aryl beta-glucoside salicin requires either salA or salB. J Bacteriol 181: 3003-3009. https://doi.org/10.1128/jb.181.10.3003-3009.1999

Finkel OM, Castrillo G, Paredes SH, Gonzalez IS, Dangl JL (2017) Understanding and exploiting plant beneficial microbes. Curr Opin Plant Biol 38 : $155-163$. https://doi.org/10.1016/j.pbi.2017.04.018

Fox SL, O'Hara GW, Brau L (2011) Enhanced nodulation and symbiotic effectiveness of Medicago truncatula when co-inoculated with Pseudomonas fluorescens WSM3457 and Ensifer(Sinorhizobium) medicae WSM419. Plant Soil 348: 245-254. https://doi.org/10.1007/s11104-011-0959-8

Fujii K, Aoki M, Kitayama K (2013) Reprint of "Biodegradation of low molecular weight organic acids in rhizosphere soils from a tropical montane rain forest". Soil Biol Biochem 56: 3-9. https://doi.org/10.1016/j.soilbio.2012.10.024. 
Ghazy N, El-Nahrawy S (2020) Siderophore production by Bacillus subtilis MF497446 and Pseudomonas koreensis MG209738 and their efficacy in controlling Cephalosporium maydis in maize plant. Arch Microbiol 203: 1195-1209. https://doi.org/10.1007/s00203-020-02113-5

Glickmann E, Dessaux Y (1995) A critical-examination of the specificity of the salkowski reagent for indolic compounds produced by phytopathogenic bacteria. Appl Environ Microbiol 61: 793-796. https://doi.org/10.1128/aem.61.2.793-796.1995

Gopalakrishnan S, Sathya A, Vijayabharathi R, Varshney RK, Gowda CLL, Krishnamurthy L (2015) Plant growth promoting rhizobia: challenges and opportunities. 3 Biotech 5: 355-377. https://doi.org/10.1007/s13205-014-0241-x

Gopi GK, Meenakumari KS, Nysanth NS, Subha P (2019) An optimized standard liquid carrier formulation for extended shelf-life of plant growth promoting bacteria. Rhizosphere 11: 100160. https://doi.org/10.1016/j.rhisph.2019.100160

Guo MJ, Shen J, Song XE, Dong SQ, Wen YY, Yuan XY, Guo PY (2019) Comprehensive evaluation of fluroxypyr herbicide on physiological parameters of spring hybrid millet. PeerJ 7: e7794. https://doi.org/10.7717/peerj.7794

Han Q, Ma Q, Chen Y, Tian B, Xu LX, Bai Y, Chen WF, Li X (2020) Variation in rhizosphere microbial communities and its association with the symbiotic efficiency of rhizobia in soybean. ISME J 14: 1915-1928. https://doi.org/10.1038/s41396-020-0648-9

Hassan MK, McInroy JA, Kloepper JW (2019) The interactions of rhizodeposits with plant growth-promoting rhizobacteria in the rhizosphere: a review. Agriculture-Basel 9: 142. https://doi.org/10.3390/agriculture9070142

Hinsinger P, Marschner P (2006) Rhizosphere - perspectives and challenges - a tribute to Lorenz Hiltner 12-17 September 2004 - Munich, Germany. Plant Soil 283: VII-VIII. https://doi.org/10.1007/s11104-006-0057-5

Huang X, Liu S, Liu X, Zhang S, Li L, Zhao H, Zhao J, Zhang J, Cai Z (2020) Plant pathological condition is associated with fungal community succession triggered by root exudates in the plant-soil system. Soil Biol Biochem 151: 108046. https://doi.org/10.1016/j.soilbio.2020.108046

Irikiin Y, Nishiyama M, Otsuka S, Senoo K (2006) Rhizobacterial community-level, sole carbon source utilization pattern affects the delay in the bacterial wilt of tomato grown in rhizobacterial community model system. Appl Soil Ecol 34: 27-32. https://doi.org/10.1016/j.apsoil.2005.12.003

Ji PS, Wilson M (2002) Assessment of the importance of similarity in carbon source utilization profiles between the biological control agent and the pathogen in biological control of bacterial speck of tomato. Appl Environ Microbiol 68: 4383-4389. https://doi.org/10.1128/aem.68.9.4383-4389.2002

Jian QJ, Li TT, Wang Y, Zhang Y, Zhao ZY, Zhang XH, Gong L, Jiang YM (2019) New insights into fumonisin production and virulence of Fusarium proliferatum underlying different carbon sources. Food Res Int 116: 397-407. https://doi.org/10.1016/j.foodres.2018.08.053.

Jung W-J, Mabood F, Souleimanov A, Park R-D, Smith DL (2008) Chitinases produced by Poenibacillus illinoisensis and Bacillus thuringiensis subsp pakistani degrade Nod factor from Bradyrhizobium japonicum. Microbiological Research 163: 345-349. https://doi.org/10.1016/j.micres.2006.06.013

Kobayashi DY, Guglielmoni M, Clarke BB (1995) Isolation of the chitinolytic bacteria Xanthomonas-maltophilia and Serratia-marcescens as biological-control agents for summer patch disease of turfgrass. Soil Biol Biochem 27: 1479-1487. https://doi.org/10.1016/0038-0717(95)00062-j

Kragelund L, Nybroe O (1996) Competition between Pseudomonas fluorescens Ag1 and Alcaligenes eutrophus JMP134 (pJP4) during colonization of barley roots. FEMS Microbiol Ecol 20: 41-51. https://doi.org/10.1016/0168-6496(96)00013-x.

Kristen MD, Eoin LB, Todd ZD, Gary LA, Steven EL, Mary KF (2009) Selective progressive response of soil microbial community to wild oat roots. ISME J 3: 168178. https://doi.org/10.1038/ismej.2008.103

Kuklinsky-Sobral J, Araujo WL, Mendes R, Geraldi IO, Pizzirani-Kleiner AA, Azevedo JL (2004) Isolation and characterization of soybean-associated bacteria and their potential for plant growth promotion. Environ Microbiol 6: 1244-1251. https://doi.org/10.1111/j.1462-2920.2004.00658.x

Kumar A, Guleria S, Mehta P, Walia A, Chauhan A, Shirkot CK (2015) Plant growth-promoting traits of phosphate solubilizing bacteria isolated from Hippophae rhamnoides L. (Sea-buckthorn) growing in cold desert Trans-Himalayan Lahul and Spiti regions of India. Acta Physiol Plant $37: 48$.

https://doi.org/10.1007/s11738-015-1793-z

Kumar GP, Kishore N, Amalraj ELD, Ahmed S, Rasul A, Desai S (2012) Evaluation of fluorescent Pseudomonas spp. with single and multiple PGPR traits for plant growth promotion of sorghum in combination with AM fungi. Plant Growth Regul 67: 133-140. https://doi.org/10.1007/s10725-012-9670-x

Lami MJ, Adler C, Santo M, Zenoff AM, de Cristobal RE, Espinosa-Urgel M, Vincent PA (2020) Pseudomonas stutzeri MJL19, a rhizosphere-colonizing bacterium that promotes plant growth under saline stress. J Appl Microbiol. https://doi.org/10.1111/jam.14692

Lebeis SL, Paredes SH, Lundberg DS, Breakfield N, Gehring J, McDonald M, Malfatti S, del Rio TG, Jones CD, Tringe SG, et al. (2015) Salicylic acid modulates colonization of the root microbiome by specific bacterial taxa. Science 349: 860-864. https://doi.org/10.1126/science.aaa8764

Lee SJ, Trxecbnh CS, Lee WJ, Jeong CY, Truong HA, Chung NY, Kang CS, Lee HJ (2020) Bacillus subtilis strain L1 promotes nitrate reductase activity in Arabidopsis and elicits enhanced growth performance in Arabidopsis, lettuce, and wheat. J Plant Res 133: 231-244. https://doi.org/10.1007/s10265-01901160-4 
Li HY, Qiu YZ, Yao T, Ma YC, Zhang HR, Yang XL (2020) Effects of PGPR microbial inoculants on the growth and soil properties of Avena sativa, Medicago sativa, and Cucumis sativus seedlings. Soil Tillage Res 199: 104577. https://doi.org/10.1016/j.still.2020.104577

Li Z, Yao Q, Chen M, Zhu H (2012) Isolating tomato rhizobacteria using root exuding pectin. Chinese Journal of Applied and Environmental Biology 18: 147152. https://doi.org/10.3724/sp.J.1145.2012.00147

Ling LL, Schneider T, Peoples AJ, Spoering AL, Engels I, Conlon BP, Mueller A, Schaberle TF, Hughes DE, Epstein S, Jones M, Lazarides L, Steadman VA, Cohen DR, Felix CR, Fetterman KA, Millett WP, Nitti AG, Zullo AM, Chen C, Lewis K (2015) A new antibiotic kills pathogens without detectable resistance. Nature 517: 455-+. https://doi.org/10.1038/nature14098.

Lugtenberg B, Kamilova F (2009) Plant-growth-promoting rhizobacteria. Annu Rev Microbiol 63: 541-556.

https://doi.org/10.1146/annurev.micro.62.081307.162918

Lunn JE, Delorge I, Figueroa CM, Van Dijck P, Stitt M (2014) Trehalose metabolism in plants. Plant J 79: 544-567. https://doi.org/10.1111/tpj.12509.

Khan MMA, Haque E, Paul NC, Khaleque MA, Al-Garni SMS, Rahman M, Islam MT (2017) Enhancement of growth and grain yield of rice in nutrient deficient soils by rice probiotic bacteria. Rice Sci 24: 264-273. https://doi.org/10.1016/j.rsci.2017.02.002.

Mahdavi SME, Salehi H, Zarei M (2020) Morpho-physiological and biochemical attributes of tall fescue (Festuca arundinacea Schreb.) inoculated with Pseudomonas fluorescens under deficit irrigation. J Soil Sci Plant Nutr 20: 1457-1471. https://doi.org/10.1007/s42729-020-00225-x

Mahdi JG (2014) Biosynthesis and metabolism of beta-d-salicin: A novel molecule that exerts biological function in humans and plants. Biotechnology Reports (Amsterdam, Netherlands) 4: 73-79. https://doi.org/10.1016/j.btre.2014.08.005

Marra LM, Soares C, de Oliveira SM, Ferreira PAA, Soares BL, Carvalho RD, de Lima JM, Moreira FMD (2012) Biological nitrogen fixation and phosphate solubilization by bacteria isolated from tropical soils. Plant Soil 357: 289-307. https://doi.org/10.1007/s11104-012-1157-z

Meyer T, Vigouroux A, Aumont-Nicaise M, Comte G, Vial L, Lavire C, Morera S (2018) The plant defense signal galactinol is specifically used as a nutrient by the bacterial pathogen Agrobacterium fabrum. J Biol Chem 293: 7930-7941. https://doi.org/10.1074/jbc.RA118.001856

Mise K, Koyama Y, Matsumoto A, Fujita K, Kunito T, Senoo K, Otsuka S (2020) Pectin drives microbial phosphorus solubilization in soil: evidence from isolation-based and community-scale approaches. Eur J Soil Biol 97: 103169. https://doi.org/10.1016/j.ejsobi.2020.103169

Monreal J, Reese ET (1969) The chitinase of Serratia marcescens. Canadian journal of microbiology 15: 689-696. https://doi.org/10.1139/m69-122

Moretti LG, Crusciol CAC, Bossolani JW, Momesso L, Garcia A, Kuramae EE, Hungria M (2020) Bacterial consortium and microbial metabolites increase grain quality and soybean yield. J Soil Sci Plant Nutr 20: 1923-1934. https://doi.org/10.1007/s42729-020-00263-5

Nordstedt NP, Jones ML (2020) Isolation of rhizosphere bacteria that improve quality and water stress tolerance in greenhouse ornamentals. Front Plant Sci 11: 826. https://doi.org/10.3389/fpls.2020.00826

Pascale A, Proietti S, Pantelides IS, Stringlis IA (2020) Modulation of the root microbiome by plant molecules: the basis for targeted disease suppression and plant growth promotion. Front Plant Sci 10: 1741. https://doi.org/10.3389/fpls.2019.01741

Patel DK, Archana G, Kumar GN (2008) Variation in the nature of organic acid secretion and mineral phosphate solubilization by Citrobacter sp DHRSS in the presence of different sugars. Curr Microbiol 56: 168-174. https://doi.org/10.1007/s00284-007-9053-0

Prasanna R, Madhan K, Singh RN, Chauhan AK, Nain L (2010) Developing biochemical and molecular markers for cyanobacterial inoculants. Folia Microbiol 55: 474-480. https://doi.org/10.1007/s12223-010-0079-5

Qin L, Jiang H, Tian J, Zhao J, Liao H (2011) Rhizobia enhance acquisition of phosphorus from different sources by soybean plants. Plant Soil 349: 25-36. https://doi.org/10.1007/s11104-011-0947-z

Radha TK, Rao DLN (2014) Plant growth promoting bacteria from cow dung based biodynamic preparations. Indian J Microbiol 54: 413-418. doi: $10.1007 /$ s12088-014-0468-6

Rahmoune B, Morsli A, Khelifi-Slaoui M, Khelifi L, Strueh A, Erban A, Kopka J, Prell J, van Dongen JT (2017) Isolation and characterization of three new PGPR and their effects on the growth of Arabidopsis and Datura plants. J Plant Interact 12: 1-6. https://doi.org/10.1080/17429145.2016.1269215.

Rajendran G, Sing F, Desai AJ, Archana G (2008) Enhanced growth and nodulation of pigeon pea by co-inoculation of Bacillus strains with Rhizobium spp. Bioresour Technol 99: 4544-4550. https://doi.org/10.1016/j.biortech.2007.06.057

Ramos-Solano B, Garcia JAL, Garcia-Villaraco A, Algar E, Garcia-Cristobal J, Manero FJG (2010) Siderophore and chitinase producing isolates from the rhizosphere of Nicotiana glauca Graham enhance growth and induce systemic resistance in Solanum lycopersicum L. Plant Soil 334: $189-197$.

https://doi.org/10.1007/s11104-010-0371-9

Page $17 / 21$ 
Rau N, Mishra V, Sharma M, Das MK, Ahaluwalia K, Sharma RS (2009) Evaluation of functional diversity in rhizobacterial taxa of a wild grass (Saccharum ravennae) colonizing abandoned fly ash dumps in Delhi urban ecosystem. Soil Biol Biochem 41: 813-821. https://doi.org/10.1016/j.soilbio.2009.01.022

Rodriguez PA, Rothballer M, Chowdhury SP, Nussbaumer T, Gutjahr C, Falter-Braun P (2019) Systems biology of plant-microbiome interactions. Mol Plant 12 : 804-821. https://doi.org/10.1016/j.molp.2019.05.006

Schwyn B, Neilands JB (1987) Universal chemical assay for the detection and determination of siderophores. Anal biochem 160:47-56. https://doi.org/10.1016/0003-2697(87)90612-9

Sharma A, Pandey A, Shouche YS, Kumar B, Kulkarni G (2009) Characterization and identification of Geobacillus spp. isolated from Soldhar hot spring site of Garhwal Himalaya, India. J Basic Microb 49: 187-194. https://doi.org/10.1002/jobm.200800194.

Shen H, Yan WH, Yang XY, He XH, Wang X, Zhang YT, Wang B, Xia QY (2020) Co-occurrence network analyses of rhizosphere soil microbial PLFAs and metabolites over continuous cropping seasons in tobacco. Plant Soil 452: 119-135. https://doi.org/10.1007/s11104-020-04560-X

Shi Y, Wang Y, Zhou Q, Liu H, Li B, Luo J, Xie G, Sun G (2012) Effect of rice root exudates and strain combination on biofilm formation of Paenibacillus polymyxa and Paenibacillus macerans. Afr J Microbiol Res 6: 3343-3347. https://doi.org/10.5897/ajmr12.246

Silini-Cherif H, Silini A, Ghoul M, Yadav S (2012) Isolation and characterization of plant growth promoting traits of a rhizobacteria: Pantoea agglomerans Ima2. Pakistan journal of biological sciences 15: 267-276. https://doi.org/10.3923/pjbs.2012.267.276

Soldan R, Mapelli F, Crotti E, Schnell S, Daffonchio D, Marasco R, Fusi M, Borin S, Cardinale M (2019) Bacterial endophytes of mangrove propagules elicit early establishment of the natural host and promote growth of cereal crops under salt stress. Microbiol Res 223: 33-43.

https://doi.org/10.1016/j.micres.2019.03.008

Spaepen S, Vanderleyden J (2011) Auxin and plant-microbe interactions. CSH Perspect Biol 3: a001438. https://doi.org/10.1101/cshperspect.a001438

Sun L, Zhu GH, Liao XY, Yan XL (2017) Interactions between Pteris vittata L. genotypes and a polycyclic aromatic hydrocarbon (PAH)-degrading bacterium (Alcaligenes sp.) in arsenic uptake and PAH-dissipation. Environ Pollut 230: 862-870. https://doi.org/10.1016/j.envpol.2017.07.037.

Suresh PV (2012) Biodegradation of shrimp processing bio-waste and concomitant production of chitinase enzyme and N-acetyl-D-glucosamine by marine bacteria: production and process optimization. World J Microbiol Biotechnol 28: 2945-2962. https://doi.org/10.1007/s11274-012-1106-2

Tabassum B, Khan A, Tariq M, Ramzan M, Khan MSI, Shahid N, Aaliya K (2017) Bottlenecks in commercialisation and future prospects of PGPR. Appl Soil Ecol 121: 102-117. https://doi.org/10.1016/j.apsoil.2017.09.030

Toscano-Verduzco FA, Cedeno-Valdivia PA, Chan-Cupul W, Hernandez-Ortega HA, Ruiz-Sanchez E, Galindo-Velasco E, Cruz-Crespo E (2020) Phosphates solubilization, indol-3-acetic acid and siderophores production by Beauveria brongniartii and its effect on growth and fruit quality of Capsicum chinense. $\mathrm{J}$ Horticult Sci Biotechnol 95: 235-246. https://doi.org/10.1080/14620316.2019.1662737

Xu Y, Wang G, Jin J, Liu J, Zhang Q, Liu X (2009) Bacterial communities in soybean rhizosphere in response to soil type, soybean genotype, and their growth stage. Soil Biol Biochem 41: 919-925. https://doi.org/10.1016/j.soilbio.2008.10.027

Valetti L, Iriarte L, Fabra A (2018) Growth promotion of rapeseed (Brassica napus) associated with the inoculation of phosphate solubilizing bacteria. Appl Soil Ecol 132: 1-10. https://doi.org/10.1016/j.apsoil.2018.08.017

Van Assche A, Alvarez-Perez S, de Breij A, De Brabanter J, Willems KA, Dijkshoorn L, Lievens B (2017) Phylogenetic signal in phenotypic traits related to carbon source assimilation and chemical sensitivity in Acinetobacter species. Appl Microbiol Biotechnol 101: 367-379. https://doi.org/10.1007/s00253-016-7866-0

Vasseur-Coronado M, du Boulois HD, Pertot I, Puopolo G (2020) Selection of plant growth promoting rhizobacteria sharing suitable features to be commercially developed as biostimulant products. Microbiol Res 245: 126672-126672. https://doi.org/10.1016/j.micres.2020.126672

Vessey JK (2003) Plant growth promoting rhizobacteria as biofertilizers. Plant Soil 255: 571-586. https://doi.org/10.1023/a:1026037216893

Vincent JM (1970) The cultivation, isolation and maintenance of rhizobia. In: Vincent JM (ed) A manual for the practical study of the root-nodule bacteria. Blackwell Scientific Publications, Oxford, pp 1-13

Walitang DI, Kim K, Madhaiyan M, Kim YK, Kang Y, Sa T (2017) Characterizing endophytic competence and plant growth promotion of bacterial endophytes inhabiting the seed endosphere of rice. BMC Microbiol 17: 209. https://doi.org/10.1186/s12866-017-1117-0

Wang F, Kang SZ, Du TS, Li FS, Qiu RJ (2011) Determination of comprehensive quality index for tomato and its response to different irrigation treatments. Agric Water Manage 98: 1228-1238. https://doi.org/10.1016/j.agwat.2011.03.004

Wang R, He CF, Dong K, Zhao X, Li YX, Hu YK (2020) Delineation of the crucial evolutionary amino acid sites in trehalose-6-phosphate synthase from higher plants. Evol Bioinform 16: 1176934320910145. https://doi.org/10.1177/1176934320910145 
Wozniak M, Galazka A, Tyskiewicz R, Jaroszuk-Scisel J (2019) Endophytic bacteria potentially promote plant growth by synthesizing different metabolites and their phenotypic/physiological profiles in the Biolog GEN III MicroPlate(TM) test. Int J Mol Sci 20: 5283. https://doi.org/10.3390/ijms20215283

Wu K, Fang ZY, Guo R, Pan B, Shi W, Yuan SF, Guan HL, Gong M, Shen B, Shen QR (2015) Pectin enhances bio-control efficacy by inducing colonization and secretion of secondary metabolites by Bacillus amyloliquefaciens SQY 162 in the rhizosphere of tobacco. PLoS One 10: e0127418.

https://doi.org/10.1371/journal.pone.0127418

Yuan J, Zhang N, Huang QW, Raza W, Li R, Vivanco JM, Shen QR (2015) Organic acids from root exudates of banana help root colonization of PGPR strain Bacillus amyloliquefaciens NJN-6. Sci Rep 5: 13438. https://doi.org/10.1038/srep13438

Zhalnina K, Louie KB, Hao Z, Mansoori N, da Rocha UN, Shi SJ, Cho HJ, Karaoz U, Loque D, Bowen BP, Firestone MK, Northen TR, Brodie EL (2018) Dynamic root exudate chemistry and microbial substrate preferences drive patterns in rhizosphere microbial community assembly. Nat Microbiol 3: $470-480$. https://doi.org/10.1038/s41564-018-0129-3

Zhang BG, Du NN, Li YJ, Shi P, Wei GH (2018) Distinct biogeographic patterns of rhizobia and non-rhizobial endophytes associated with soybean nodules across China. Sci Total Environ 643: 569-578. https://doi.org/10.1016/j.scitotenv.2018.06.240.

Zhang BH, Hong JP, Zhang Q, Jin DS, Gao CH (2020) Contrast in soil microbial metabolic functional diversity to fertilization and crop rotation under rhizosphere and non-rhizosphere in the coal gangue landfill reclamation area of Loess Hills. PLoS One 15: e0229341.

https://doi.org/10.1371/journal.pone.0229341

Zhang J, Wang PC, Fang L, Zhang QA, Yan CS, Chen JY (2017) Isolation and characterization of phosphate-solubilizing bacteria from mushroom residues and their effect on tomato plant growth promotion. Pol J Microbiol 66: 57-65. https://doi.org/10.5604/17331331.1234993

Zhang ZQ, Xu Y, Song GM, Gao XY, Zhao YQ, Jia MZ, Chen YF, Suo B, Chen QM, Wu D, Wu WX, Wen JZ (2019) Phytophthora sojae zoospores differ in chemotaxis to the root and root exudates of host soybean and nonhost common bean. J Gen Plant Pathol 85: 201-210. https://doi.org/10.1007/s10327-01900839-9

Zhao LF, Deng ZS, Yang WQ, Cao Y, Wang ET, Wei GH (2010) Diverse rhizobia associated with Sophora alopecuroides grown in different regions of Loess Plateau in China. Syst Appl Microbiol 33: 468-477. https://doi.org/10.1016/j.syapm.2010.08.004

\section{Figures}




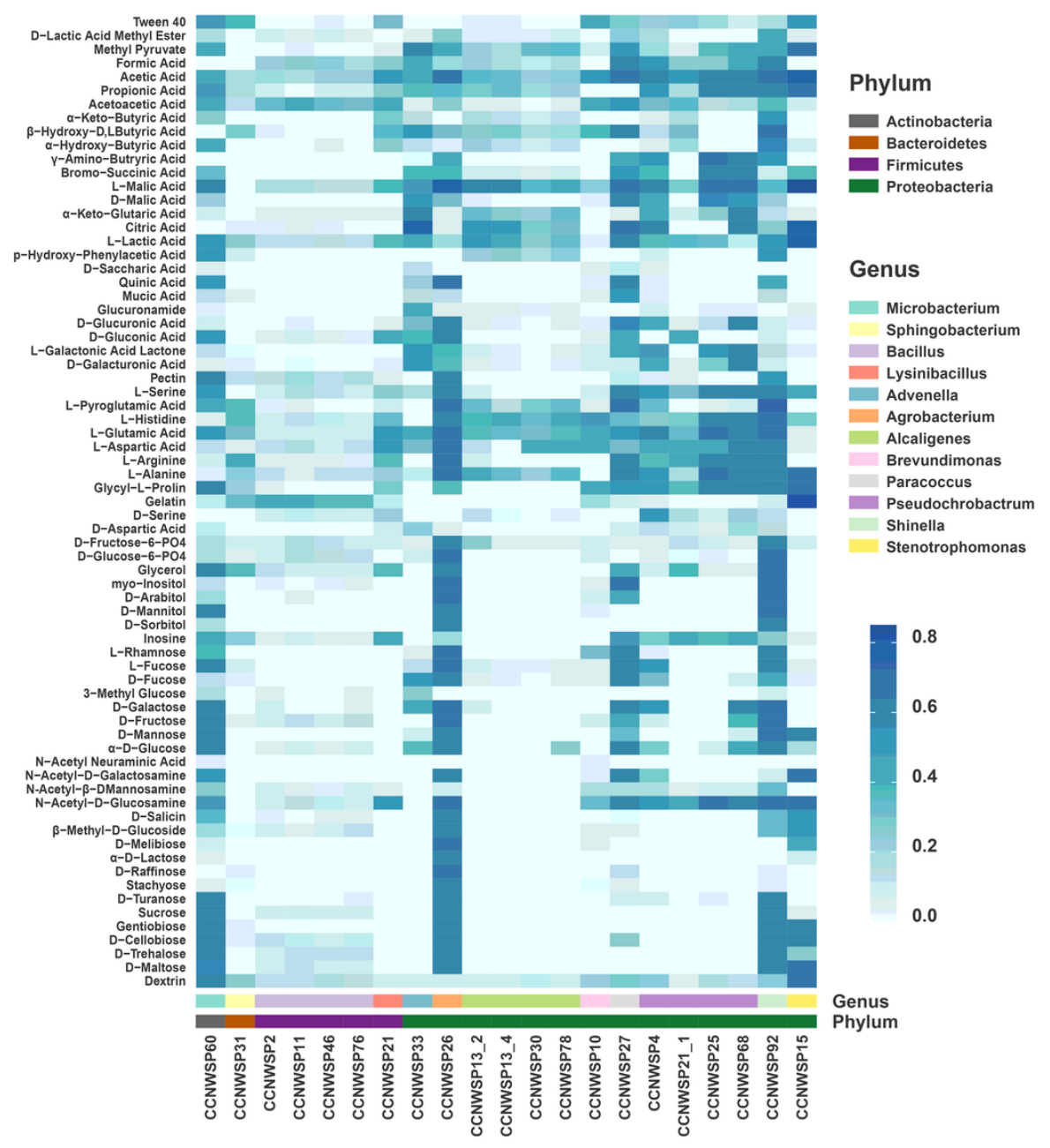

\section{Figure 1}

Well Color Development values of 21 rhizobacterial isolates, representing their ability to metabolize 71 carbon substrates in Biolog Genlll microplates. The data are the mean values $(n=2)$. 


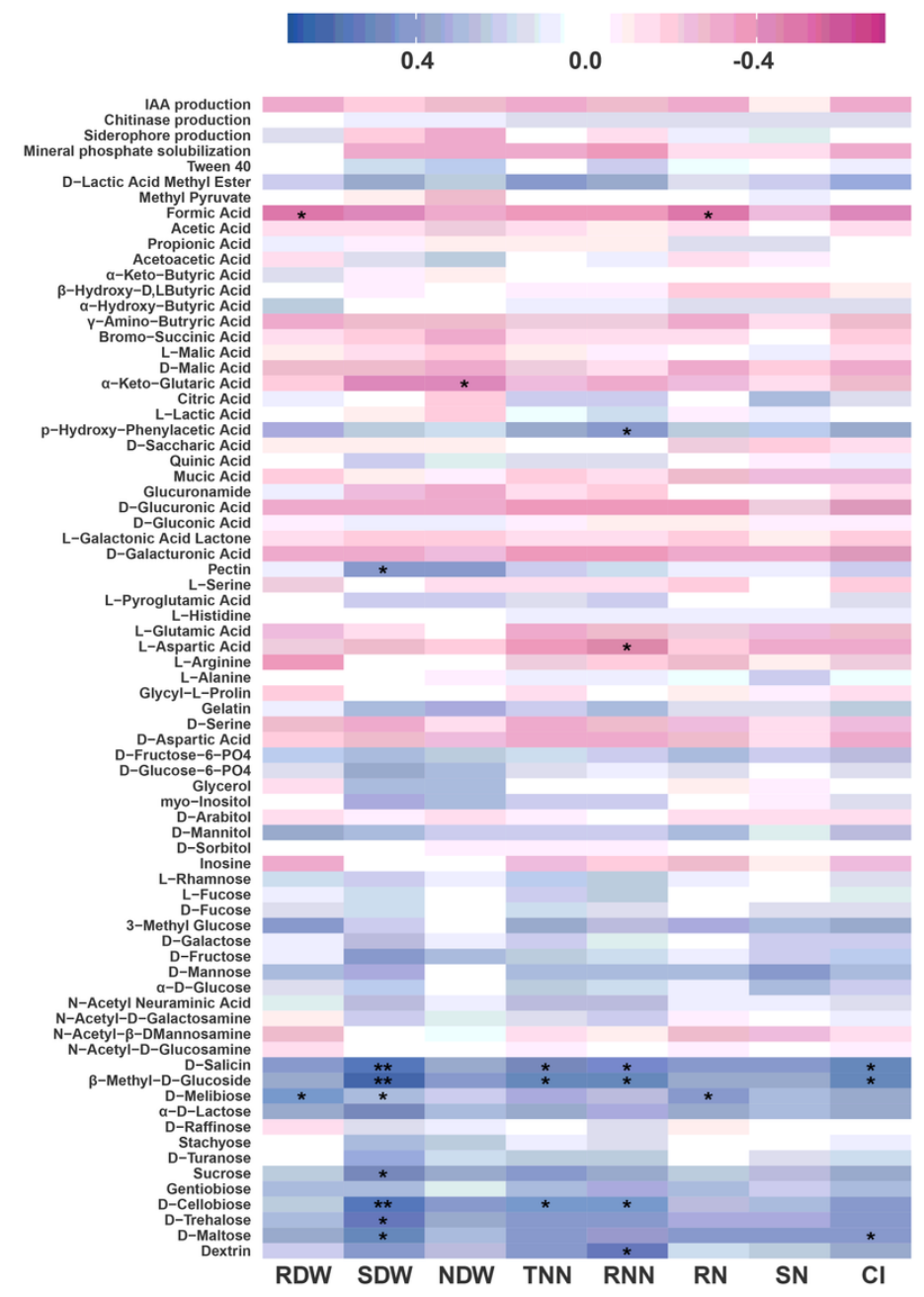

\section{Figure 2}

Spearman's correlation coefficients between the metabolic phenotypes of the rhizobacterial isolates and their promotional effects on the soybean-rhizobium system. RDW, root dry weight; SDW, shoot dry weight; NDW, dry weight per nodule; TNN, total number of nodules; RNN, number of red nodules; RN, root total nitrogen; SN, shoot total nitrogen; and Cl, comprehensive index. *, Significant correlation at $p<0.05$ and **, significant correlation at $p<0.01(n=21)$.

\section{Supplementary Files}

This is a list of supplementary files associated with this preprint. Click to download.

- ESM1.xIsx

- ESM2.xIsx

- ESM3.xIsx

- ESM4.xlsx 